\title{
Magnetic MAX phases from theory and experiments; a review
}

\author{
Arni Sigurdur Ingason, Martin Dahlqvist and Johanna Rosén \\ Journal Article
}

\section{Tweet}

N.B.: When citing this work, cite the original article.

Original Publication:

Arni Sigurdur Ingason, Martin Dahlqvist and Johanna Rosén, Magnetic MAX phases from theory and experiments; a review, Journal of Physics, 2016. 28(43), pp.433003.

http://dx.doi.org/10.1088/0953-8984/28/43/433003

Copyright: IOP Publishing: Hybrid Open Access

http://www.iop.org/

Postprint available at: Linköping University Electronic Press

http://urn.kb.se/resolve?urn=urn:nbn:se:liu:diva-132203

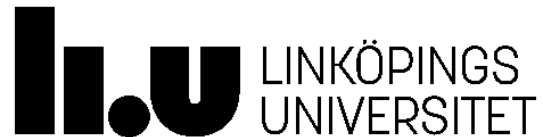




\title{
Magnetic MAX phases from theory and experiments; A review
}

\author{
A.S. Ingason, ${ }^{1, *}$ M. Dahlqvist,${ }^{1}$ and J. Rosen ${ }^{1}$ \\ ${ }^{1}$ Thin Film Physics, Department of Physics, Chemistry and Biology (IFM), \\ Linköping University, SE-581 83 Linköping, Sweden
}

(Dated: October 8, 2016)

\begin{abstract}
This review presents MAX phases ( $M$ is a transition metal, $A$ an A-group element, $X$ is $\mathrm{C}$ or $\mathrm{N}$ ), known for their unique combination of ceramic/metallic properties, as a recently uncovered family of novel magnetic nanolaminates. The first created magnetic MAX phases were predicted using density functional theory through evaluation of phase stability, and subsequently synthesized as heteroepitaxial thin films. All magnetic MAX phases reported to date, in bulk or thin film form, are based on $\mathrm{Cr}$ and/or $\mathrm{Mn}$, and they include $(\mathrm{Cr}, \mathrm{Mn})_{2} \mathrm{AlC},(\mathrm{Cr}, \mathrm{Mn})_{2} \mathrm{GeC},(\mathrm{Cr}, \mathrm{Mn})_{2} \mathrm{GaC}$, $(\mathrm{Mo}, \mathrm{Mn})_{2} \mathrm{GaC}$, and $(\mathrm{V}, \mathrm{Mn})_{3} \mathrm{GaC}_{2}, \mathrm{Cr}_{2} \mathrm{AlC}, \mathrm{Cr}_{2} \mathrm{GeC}$ and $\mathrm{Mn}_{2} \mathrm{GaC}$. A variety of magnetic properties have been found, such as ferromagnetic response well above room temperature and structural changes linked to magnetic anisotropy. In this paper, theoretical as well as experimental work performed on these materials to date is critically reviewed, in terms of methods used, results acquired, and conclusions drawn. Open questions concerning magnetic characteristics are discussed, and an outlook focused on new materials, superstructures, property tailoring and further synthesis and characterization is presented.
\end{abstract}

PACS numbers: 81.15.Cd, 81.15.Lm, 75.70.Ak, 75.30.Kz

\section{INTRODUCTION}

The $M_{n+1} A X_{n}$ (MAX) phases $(n=1-3)$ are a family of atomically laminated materials where $M$ is a transition metal, $A$ an A-group element, and $X$ refers to carbon and/or nitrogen see Fig. 1. After being first synthesized in the 1960s [1], they were rediscovered in the 1990s when Barsoum et al. revealed a unique combination of ceramic and metallic properties [2]. Since then, they have been routinely synthesized as bulk and as thin films, and have turned out to exhibit exceptional physical, chemical, electrical and mechanical properties [2]. These stem from the inherently laminated structure, and a mixture of strong M-X bonds and weaker M-A bonds.

A fascinating aspect of working with MAX phases is the large number of possible isostructural compositions. This ensures a rapid progress in understanding the role of chemistry on the material properties. Experimental evidence of magnetic MAX phases were first reported in $2013[3,4]$, and even though it is still a field in its infancy, the results presented to date indicate a plethora of potential magnetic ground states and related properties. Their laminated character also make them an ideal model system for the study of the complex magnetic phenomena that occur in atomically layered materials, extending beyond traditional ferromagnetic (FM), antiferromagnetic (AFM), and paramagnetic $(\mathrm{PM})$ order.

Potential applications mentioned to date range from spintronics to refrigeration, even though the research efforts have so far been focused solely on the discovery of new magnetic phases and compositions, and

\footnotetext{
*arnsi@ifm.liu.se
}

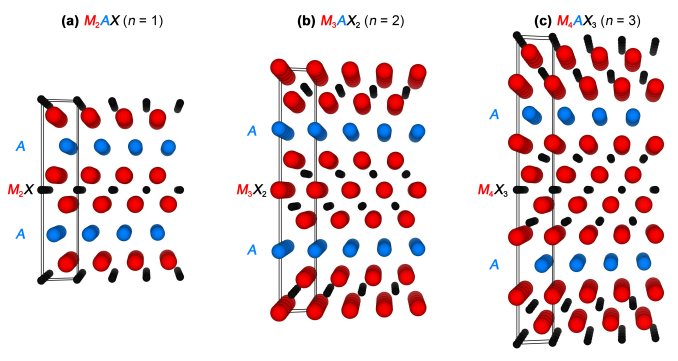

Figure 1. A schematic illustrating the general crystal structure of the $M_{n+1} A X_{n}$ phases for $n=1-3$.

fundamentals of magnetic properties. In the present paper we will review the methods used for calculations, synthesis and characterization of MAX phases and the obtained results thereof where there is a focus on, at least in part, the magnetic characteristics. All materials and related reports are listed in Table I. Section 2 covers theoretical investigations performed on known and hypothetical magnetic MAX phases, with a particular emphasis on electron correlation effects, phase stability, spin configurations and magnetic ground state identification. This is followed by a review of the methods used for materials synthesis in Section 3, both in bulk as well as in thin film form, the obtained materials, and the results from their characterization.

Even though it is not straightforward to summarize general magnetic characteristics of these materials, we elaborate on the effect of sample quality and materials composition on the magnetic properties in Section 4. We also address the question of local or itinerant magnetism, as well as the possibility of collinear or non-collinear spin configurations. In Section 5, we present an outlook for this relatively new research area, including potential 
tuning of the MAX phase properties, further techniques for evaluation thereof, and general future research routes.

\section{STABILITY AND MAGNETISM FROM THEORETICAL INVESTIGATIONS}

\section{A. Methods}

First principles calculations within the framework of density functional theory (DFT) $[42,43]$ is a commonly used tool for materials research [44]. Much theoretical work on MAX phases has been performed using DFT within the local density approximation (LDA) or the generalized gradient approximation (GGA). However, one should bear in mind that in general LDA tends to overestimate bond strength and underestimate lattice parameters, while GGA often results in too large lattice parameters in comparison with measured values, with the exception of $\mathrm{Cr}$ - and Mn-based MAX phases. GGA exists in many different forms [45-47], which are based on different approximations, where the parametrization by Perdew, Burke, and Ernzerhof (PBE) is most commonly used for calculations of MAX phases. Correspondingly, typical software used for performing electronic structure calculations are Vienna Ab initio Simulation Package (VASP), Cambridge Serial Total Energy Package (CASTEP), and WIEN2K. Both VASP and CASTEP use plane-wave basis sets for describing valence electrons whereas core electrons are treated as frozen using different pseudopotentials. WIEN2K is based on the full-potential linearized augmented plane-wave (FLAPW) method which includes all electrons. It is among the most accurate schemes for electronic structure calculations but requires more computational power and is more time consuming.

Density functional theory calculations within LDA and GGA are useful for calculating ground-state properties of materials with weak electronic correlations. However, for strongly correlated materials, e.g., actinides or middle-to-late transition metal oxides and nitrides, the large Coulombic repulsion between localized electrons might be overestimated [48-54]. This results in an inaccurate description of relative energies, magnetic ground states, and electronic structure, and may even convert what should be an insulator to a metal. For such materials the many electrons in partially filled $d$ or $f$ orbitals are inherently localized on each metal atom and by introducing the on-site Coulomb repulsion U, applied to localized electrons such as $3 d$ or $4 f$, i.e., a DFT+U approach, results are improved [55-58]. Two commonly used methods for treating strongly correlated electron materials, are through empirical fitting or constrained DFT calculations [59-62]. The former method lacks a theoretical basis and is influenced by a limited or non-existing amount of experimental data, whereas the latter approach is motivated from first-principles but of questionable accuracy due to the artificial nature of the constrained system [54].

The use of DFT $+\mathrm{U}$ methods in calculation of MAX phases was first introduced for $\mathrm{Cr}_{2} \mathrm{AlC}[8,9]$ primarily motivated by three observations; (i) the reported number of states at the Fermi level $\mathrm{N}\left(\mathrm{E}_{\mathrm{f}}\right)$ for $\mathrm{Cr}_{2} \mathrm{AlC}$ is among the highest of all $M_{2} \mathrm{AlC}$ phases (calculated $6.3-8.0$ and measured 12.9 - 14.5 states / eV unit cell) while the measured electrical conductivity is among the lowest [5, 6, 63-65], (ii) $\mathrm{Cr}_{2} \mathrm{AlC}$ has been found to have a much higher calculated bulk modulus ( $>200 \mathrm{GPa}$ ) as compared to measured values (138 to $165 \mathrm{GPa}$ ) $[5,6,64,66,67]$, and (iii) an underestimated calculated equilibrium volume of about $-1.5 \%$ in comparison to experiment $[5,6,65,67,68]$. By using DFT+U, Du et al. [8] and Ramzan et al. [9] were able to stabilize a ferromagnetic state of $\mathrm{Cr}_{2} \mathrm{AlC}$ for an increased equilibrium volume, 90.3 and $90.62 \AA^{3} /$ unit cell, respectively, with a resulting decrease in bulk modulus, 164 and $182 \mathrm{GPa}$, as compared to conventional DFT, i.e., $\mathrm{U}=0$. Du et al. also found a decrease in the DOS at the Fermi level, to $\mathrm{N}\left(\mathrm{E}_{\mathrm{f}}\right)=2.38$ states $/ \mathrm{eV}$ unit cell [8]. Later, Dahlqvist et al. suggested that the theoretical and experimental inconsistency, allegedly solved by introducing $+\mathrm{U}$, can be related to considered magnetic spin configurations [10]. By expanding the $\mathrm{Cr}_{2} \mathrm{AlC}$ unit cell in-plane and considering an in-plane AFM spin configuration, the bulk modulus was decreased from 188 (non magnetic (NM)) to $171 \mathrm{GPa}$ (in-plane AFM) while the unit cell volume increased from 89.0 to $89.5 \AA^{3}$. Later, it was demonstrated that a value of $\mathrm{U}>$ $1 \mathrm{eV}$ results in structural parameters deviating strongly from experimentally observed values [13]. Tables II and III summarize theoretical and experimental data for $\mathrm{Cr}_{2} A \mathrm{C}(A=\mathrm{Al}, \mathrm{Ge}, \mathrm{Ga})$ and Mn-containing phases including lattice parameters, unit cell volumes, bulk modulus, number of states at the Fermi level, and information related to magnetism. 
Table I. All known and hypothetical MAX phases that have been discussed to date with respect to magnetism.

\begin{tabular}{|c|c|c|c|c|}
\hline Material & Theory & Bulk synthesis & Thin film synthesis & Magnetic behaviour studied \\
\hline $\mathrm{Cr}_{2} \mathrm{AlC}$ & {$[5-14]$} & {$[15]$} & {$[6,16]$} & {$[15,16]$} \\
\hline $\mathrm{Cr}_{2} \mathrm{GeC}$ & {$[3,13,17-19]$} & {$[20]$} & {$[16]$} & {$[16,20]$} \\
\hline $\mathrm{Cr}_{2} \mathrm{GaC}$ & {$[13,21,22]$} & {$[4,21]$} & {$[22]$} & {$[4,21]$} \\
\hline $\mathrm{Cr}_{2} \mathrm{AlN}$ & {$[14]$} & & & \\
\hline $\mathrm{Cr}_{2} \mathrm{GaN}$ & {$[21,23]$} & {$[21]$} & & {$[21,23]$} \\
\hline $\mathrm{Fe}_{n+1} A \mathrm{C}_{n}(A=\mathrm{Al}, \mathrm{Si}, \mathrm{Ge}, n=1-3)$ & {$[24]$} & & & \\
\hline $\mathrm{Mn}_{2} \mathrm{AlC}$ & {$[7,14]$} & & & \\
\hline $\mathrm{Mn}_{2} \mathrm{GaC}$ & {$[25-29]$} & & {$[25,27]$} & {$[25,27]$} \\
\hline$(\mathrm{Cr}, \mathrm{Mn})_{2} \mathrm{AlC}$ & {$[7,12]$} & {$[30]$} & {$[12,31]$} & {$[31]$} \\
\hline$(\mathrm{Cr}, \mathrm{Mn})_{2} \mathrm{GeC}$ & {$[3]$} & {$[20,32]$} & {$[3]$} & {$[3,20,32]$} \\
\hline$(\mathrm{Cr}, \mathrm{Mn})_{2} \mathrm{GaC}$ & & {$[4,30]$} & {$[33,34]$} & {$[4,30,33,34]$} \\
\hline$(\mathrm{Mo}, \mathrm{Mn})_{2} \mathrm{GaC}$ & & & {$[35]$} & {$[35]$} \\
\hline$(\mathrm{V}, \mathrm{Mn})_{3} \mathrm{GaC}_{2}$ & & & {$[36]$} & {$[36]$} \\
\hline $\mathrm{Cr}_{4} \mathrm{AlN}_{3}$ & {$[37]$} & & & \\
\hline$\left(\mathrm{Cr}_{1 / 3} \mathrm{Ti}_{2 / 3}\right)_{3} \mathrm{AlC}_{2}$ & {$[38,39]$} & {$[38,40,41]$} & & \\
\hline
\end{tabular}




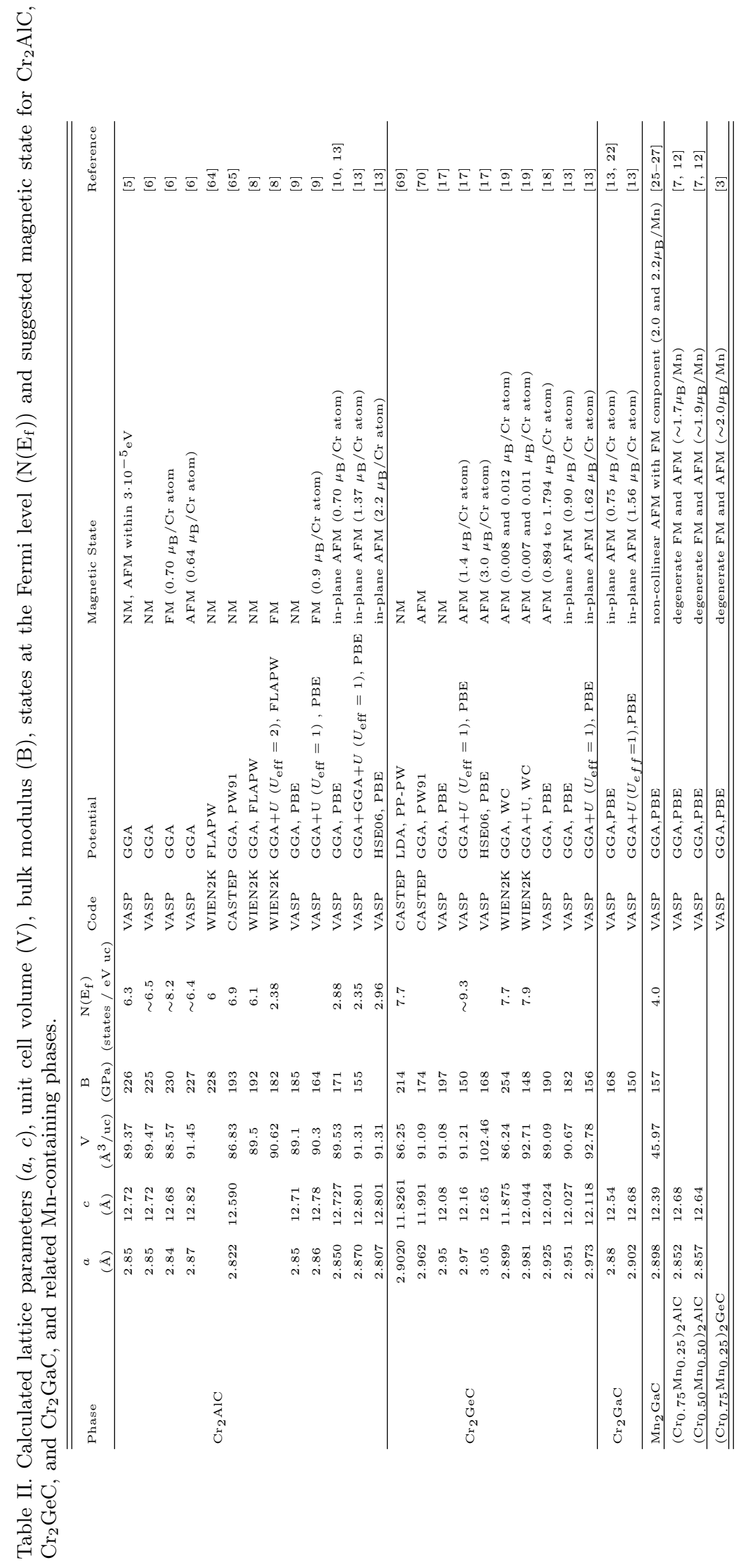




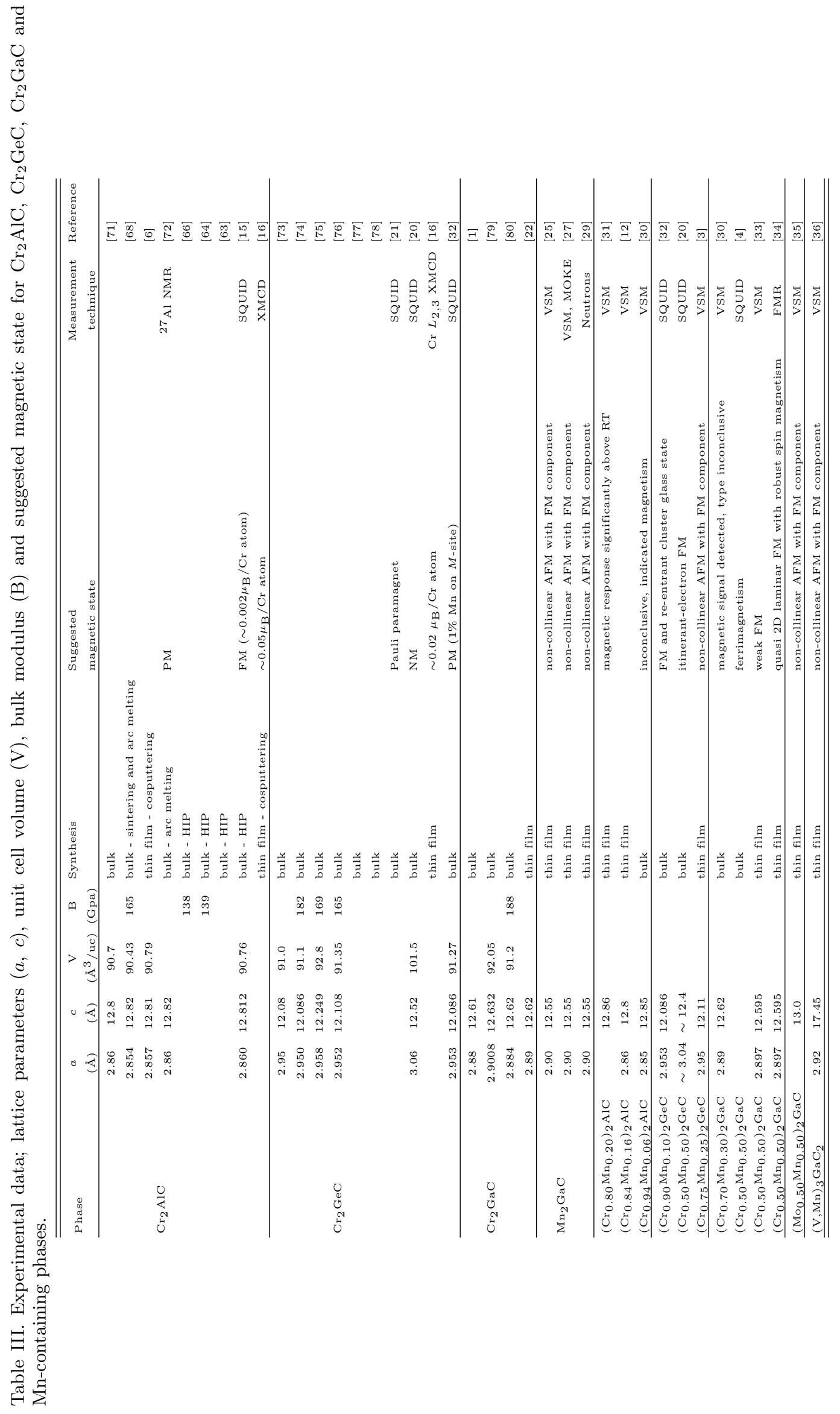




\section{B. Phase stability}

Evaluation of phase stability is a powerful tool for the prediction of new phases, as demonstrated for several well-known ternary carbide and nitride MAX phases $[14,81]$. In particular, the first magnetic MAX phases were predicted using DFT and evaluation of phase stability and subsequently synthesized [3, 7, 12, 25]. The most successfull approach that has been used for evaluating the stability of a MAX phase of composition $M_{n+1} A X_{n}$, defined as the formation enthalpy per atom $\Delta H\left(M_{n+1} A X_{n}\right)$, is to calculate the energy difference with respect to a set of competing phases,

$\Delta H\left(M_{n+1} A X_{n}\right)=\frac{E_{0}\left(M_{n+1} A X_{n}\right)-E_{0}(\text { competing phases })}{2(n+1)}$

where $E_{0}\left(M_{n+1} A X_{n}\right)$ is the total energy of a $M_{n+1} A X_{n}$ phase, and $\mathrm{E}_{0}$ (competing phases) is the total energy for a set of competing phases with total stoichiometry equal to $M_{n+1} A X_{n}$. If $\Delta H\left(M_{n+1} A X_{n}\right)<0$, the $M_{n+1} A X_{n}$ phase is considered to be stable and if $\Delta H\left(M_{n+1} A X_{n}\right)>$ 0 it is not. The consistency between theoretical predictions and experimental verification based on the constraint that the MAX phase free energy is primarily governed by the $0 \mathrm{~K}$ energy terms, $E_{0}$, [14] has been given a plausible explanation by Thore et al. [82] showing that temperature dependent terms, i.e., electronic and vibrational free energies, cancel each other out.

The first magnetic MAX phases, suggested by theory were Fe-based [24], including $\mathrm{Fe}_{2} \mathrm{AlC}$ and $\mathrm{Fe}_{2} \mathrm{SiC}$. These phases were concluded to be antiferromagnetic (AFM) with $\Delta \mathrm{H}_{\mathrm{cp}}=-181 \mathrm{meV} /$ atom and $-110 \mathrm{meV} /$ atom compared to $\mathrm{Al}_{4} \mathrm{C}_{3}, \mathrm{Fe}_{3} \mathrm{C}$ and $\mathrm{Fe}$ (for $\mathrm{Fe}_{2} \mathrm{AlC}$ ) and $\mathrm{SiC}$ and $\mathrm{Fe}$ (for $\mathrm{Fe}_{2} \mathrm{SiC}$ ). However, experimental evidence of their existence is still not reported. This was later suggested to be related to the choice of competing phases, e.g., lack of the inverse perovskite $\mathrm{Fe}_{3} \mathrm{AlC}$. By including these phases, $\mathrm{Fe}_{2} \mathrm{AlC}$ was found to be at best metastable, with $\Delta \mathrm{H}_{\mathrm{cp}}=+116 \mathrm{meV} /$ atom [7]. The first predicted stable magnetic MAX phase [7] to be synthesized was $\left(\mathrm{Cr}_{1-x} \mathrm{Mn}_{x}\right)_{2} \mathrm{AlC}$ [12]. Theoretically, a chemically ordered structure with Mn-C-Mn-Al-Cr-C-Cr-Al layer stacking along the $c$-direction was identified as the low energy structure, with FM ordered Mn layers coupled via nearly nonmagnetic Cr layers [7]. The degree of intermixing between $\mathrm{Cr}$ and $\mathrm{Mn}$ layers was also addressed as a possible route to tune the sign and magnitude of the coupling. Later theoretical predictions include $\left(\mathrm{Cr}_{1-x} \mathrm{Mn}_{x}\right)_{2} \mathrm{GeC}$, which was shown to be stabilized by $\mathrm{Cr} / \mathrm{Mn}$ disorder and related configurational entropy upon an increased temperature. At $900 \mathrm{~K}$ and for $x$ $=0.25$, the phase is predicted stable with a Gibbs free energy of $-5 \mathrm{meV} /$ atom. Common for simulated MAX phase alloys based on $\mathrm{Cr}$ and $\mathrm{Mn}$ is that at $0 \mathrm{~K}$ a chemically ordered distribution of $\mathrm{Cr}$ and $\mathrm{Mn}$ on the $M$-site is favoured. However, at moderate temperatures around $\sim 600 \mathrm{~K}$ the chemically disordered alloy is favoured due to configurational entropy. In particular, this applies to $M_{2} A X$ phases. On the contrary, $\left(\mathrm{Ti}_{1 / 3} \mathrm{Cr}_{2 / 3}\right){ }_{3} \mathrm{AlC}_{2}[38,40]$ and $\left(\mathrm{V}_{1 / 3} \mathrm{Cr}_{2 / 3}\right)_{2} \mathrm{AlC}_{3}$ [83] are examples of recent discoveries of chemically ordered quaternary MAX phases, with atomic layers composed of a single element only. Even though they contain $\mathrm{Cr}$, magnetic characteristics have to date only been explored theoretically [39]. For ternary magnetic MAX phases other than $\mathrm{Cr}_{2} A \mathrm{C}$ with $A=\mathrm{Al}$, Ge, Ga, the first synthesized phase was with $\mathrm{Mn}$ as sole $M$ element, $\mathrm{Mn}_{2} \mathrm{GaC}$, based on guidance from stability calculations with $\Delta \mathrm{H}=-30 \mathrm{meV} /$ atom.

Phase stability calculations at $0 \mathrm{~K}$ have been proven successful when it comes to verify existing $[12,14,22,26$, 81, 84-86] and, more importantly, predicting new MAX phases and related materials [3, 7, 25, 87]. When it comes to magnetic MAX phases, almost all calculations to date neglect effects from temperature. Available approaches to address such effects within DFT are the temperature dependent effective potential (TDEP) method which uses ab initio molecular dynamics (AIMD) including both lattice and electronic entropy [88], and the disordered local moment method in combination with molecular dynamics (DLM-MD) for modelling the paramagnetic state [89].

\section{Identification of magnetic ground-state}

In order to identify the magnetic ground-state, different magnetic spin configurations need to be considered. In the literature, the considered magnetic spin configurations for magnetic MAX phases are typically FM (parallel spin orientation) and AFM (antiparallel spin orientation). The considered AFM spin configurations are often of one type and generally undefined although one may assume that they consist of alternating spin up and spin down $M$ layers along the $c$-axis, i.e., AFM[0001 $]_{1}[6,8,9,11,17-19,24,37]$. The majority of theoretically investigated magnetic MAX phases are $M_{2} A X$ phases.

For $\mathrm{Cr}_{2} A \mathrm{C}$ with $A=\mathrm{Al}$, Ge, Ga, the various suggestions for the magnetic ground-state diverge. This is at least in part explained by the different simulated AFM structures within the unit cell of $\mathrm{Cr}_{2} A \mathrm{C}$ phases being limited, as there are only four $\mathrm{Cr}$ atoms distributed in four layers. Calculations performed with such size constraint may lead to a NM solution, with Cr moments of zero, even though the initial spin structure was set to FM or AFM [10] or to ferrimagnetic solutions, as suggested by Mattesini and Magnuson [19] and Li et al. [18] for $\mathrm{Cr}_{2} \mathrm{GeC}$. An alternative approach for the search of ground states is to extend the unit cell both in- and out-of-plane and hence allow for more complex AFM ordering. Such examples can be found in Ref. [10] where in-plane AFM spin configurations were considered for $\mathrm{Cr}_{2} \mathrm{AlC}$, and found to decrease the energy of the system. A systematic expansion of the unit cell out-of-plane for 


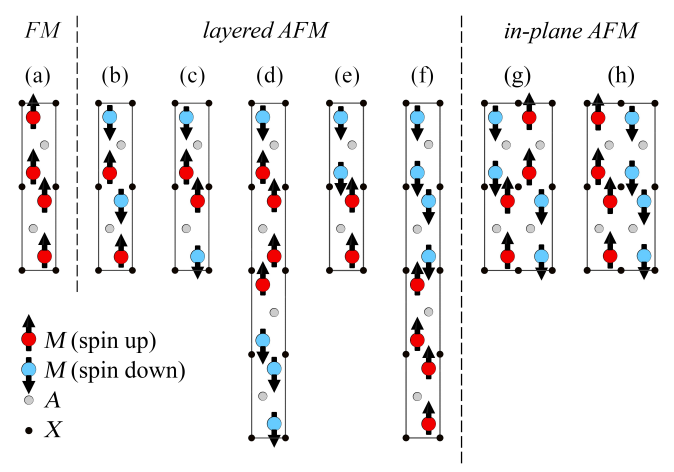

Figure 2. Schematic illustration for a selection of possible ordered collinear magnetic spin configurations for $M_{2} A X$, representing (a) FM , (b) $\operatorname{AFM}[0001]_{1}$ (c) $\mathrm{AFM}[0001]_{2}^{A}$, (d) $\operatorname{AFM}[0001]_{4}^{A}, \quad$ (e) $\operatorname{AFM}[0001]_{2}^{X}$, (f) $\operatorname{AFM}[0001]_{4}^{X}, \quad(g)$ in-AFM1, and (h) in-AFM2 order. The projections are viewed along the $[1 \overline{2} 10]$ direction. The notation used to describe the different AFM spin configurations was introduced in Ref. [10] and later extended in [27].

$\mathrm{Mn}_{2} \mathrm{GaC}$ resulted in additional spin configurations of lower energy as compared to those possible to model within the 8 atom unit cell [27]. Figure 2 illustrates a selection of possible collinear spin configurations used to model for magnetic $M_{2} A X$ phases, partly taken from Ref. [27]. An approach to find more complex and maybe less intuitive spin configurations was demonstrated in Ref. [27] where a magnetic ground-state search was performed using Heisenberg Monte Carlo simulations within the canonical ensemble using magnetic exchange interaction (MEI) parameters between different $\mathrm{Mn}$ layers. For small volumes FM ordering was found, though with increasing volume, $\mathrm{Mn}_{2} \mathrm{GaC}$ attained a spin-wave like configuration with a small net moment before reaching different non-collinear AFM solutions.

A paramagnetic state is when the atoms have non-zero moment but with a random spin orientation. Modelling paramagnetic phases of magnetic materials as non-magnetic could lead to erroneous conclusions [90]. In most materials, local magnetic moments do survive above the magnetic transition temperature, and hence a proper treatment of magnetic disorder is essential for the predictive description and exploration of materials properties, especially close to the order-disorder transition temperature, the Curie $\mathrm{T}_{\mathrm{C}}$ or Néel $\mathrm{T}_{\mathrm{N}}$ for $\mathrm{FM}$ and AFM materials, respectively [91]. An approach used to model the PM state of MAX phases [7, 10, 28] is the disordered local moments (DLM) method [92] where a solid solution with $50 \%$ up and $50 \%$ down spins on the $M$ sublattice is simulated using the special quasi-random structures (SQS) method [93]. For Mn-containing MAX phases, such as $\left(\mathrm{Cr}_{0.5} \mathrm{Mn}_{0.5}\right)_{2} \mathrm{AlC}$ [7] and $\mathrm{Mn}_{2} \mathrm{GaC}$ [28], the DLM method works with sustained disorder and non-zero local magnetic moments after electronic relaxation. Attempts to model paramagnetic $\mathrm{Cr}_{2} \mathrm{AlC}$ by means of DLM has resulted in a semi-ordered spin state [10]. This indicates that alternative methods to describe the paramagnetic state of Cr-rich MAX phases should be considered. One such approach, which accounts for correlation effects, would be a combination of DFT with model treatments of many-body effects, such as the Hubbard model, e.g., within the dynamical mean-field theory (DMFT) [94]. Transition temperatures have been explored in a recent paper by Thore et al. [28] where the magnetic critical order-disorder temperature was predicted for $\mathrm{Mn}_{2} \mathrm{GaC}$. By using a first-principles approach based on supercell calculations, the exchange interaction parameters $J_{i j}$ between pairs of Mn atoms on sites $i$ and $j$ of the bilinear Heisenberg Hamiltonian were derived using the novel magnetic direct cluster averaging method. The $J_{i j}$ 's were then used as input for Monte Carlo simulations, and in turn used to derive the magnetic energy, specific heat, and $\mathrm{T}_{\mathrm{C}}$. For $\mathrm{Mn}_{2} \mathrm{GaC}$, $\mathrm{T}_{\mathrm{C}}$ was estimated to be $660 \mathrm{~K}$, a value which remains to be experimentally verified.

\section{MATERIALS SYNTHESIS}

\section{A. Synthesis methods}

Generally MAX phases are synthesized using bulk methods such as pressureless sintering, hot isostatic pressing (HIP), self-propagating high-temperature synthesis, pulse discharge sintering and solid-liquid reaction synthesis [2]. Bulk methods yield polycrystalline samples of varying phase purity although phase pure samples are not uncommon. Recently very large (area of a few $\mathrm{mm}^{2}$ ) single crystal samples have been obtained from high temperature solution growth [95]. The synthesis of MAX phase thin films deposited using chemical vapor deposition (CVD) was first reported in 1987 by Goto and Hirai [96] and in 2002 the first report of MAX phase synthesis using physical vapor deposition (PVD) was published [97]. With the bulk synthesis of MAX phases thoroughly established and the rapid growth of synthesis using PVD [98], detailed studies on the fundamentals of these materials can be realized. As mentioned above, magnetic MAX phases are generally made by alloying a known MAX phase, most commonly $\mathrm{Cr}$ based, with a magnetic element, e.g. Mn. Synthesis of such samples has been reported both for bulk $[4,15,20,21,30,32]$ as well as for thin films $[3,12,16,31,33-36]$, see Table III. Typically the temperature during bulk synthesis can reach $1400{ }^{\circ} \mathrm{C}$ while for PVD it is normally between $450-650{ }^{\circ} \mathrm{C}$.

\section{Bulk Synthesis}

Bulk synthesis is a method allowing high quality, single phase samples of a given material. If done correctly the samples will be polycrystalline with a relaxed structure 
of relatively large crystallites that lends itself to detailed analysis with methods that require a large sample volume. This allows for the structure to be evaluated completely as opposed to for textured or epitaxial thin films which due to their orientation and small volume make analysis more complicated.

Generally this type of synthesis of MAX phases starts with the mixing of the elements that constitute the phase in the expected ratios. This mixture is then enclosed in an evacuated container, usually graphite or quartz, and then heated for extended periods of time (up to several days). The resulting sample is then ground down to a powder, pressed into a pellet and then heated again in order to achieve at a uniform sample. The same procedure is used when adding $\mathrm{Mn}$ to the mixture where the $\mathrm{Cr}$ is reduced by the amount of $\mathrm{Mn}$ to be incorporated. Commonly the resulting powder is then analyzed using X-ray diffraction (XRD) and if no appreciable amount of other phases is found in the diffractogram the sample is assumed to have the composition of the virgin materials. This is sometimes supported by looking closely at how the diffraction peaks change as a function of Mn content where it is assumed that due to the smaller atomic radius of $\mathrm{Mn}$ the lattice will shrink. This is however, an assumption which is not always supported by theory and results from different studies, including experiments, do not agree that this is the case. Additionally, the density of the samples is measured and compared to calculated values. Rietveld refinement is commonly used to confirm the structure as well as to obtain accurate measurements of the lattice parameters, and providing a qualitative measure of the amount of other phases detected. This general methodology is employed for all the bulk produced samples discussed here $[4,15,20,21,30,32]$. In Ref [30] the composition of the resulting powders was analyzed further using both energy dispersive X-ray analysis (EDX) in a scanning electron microscope (SEM) as well as EDX in a transmission electron microscope (TEM).

\section{Thin Film synthesis}

Since 2002 [97] MAX phases have been increasingly synthesized as thin film materials for potential applications such as oxidation-resistant and protective coatings on, e.g., turbine blades [99], bond coatings on sapphire fibers [100], and low friction and oxidation-resistant contacts [101]. In the latter examples the focus is primarily on achieving single-phase films of high density, where steps towards making high quality single crystal samples have mostly been taken since 2010. A routine method to synthesize these materials in thin film form is PVD, such as cathodic arc [102], or, most often, magnetron sputtering [98, 103, 104] which, combined with a suitable substrate and co-deposition from elemental targets at a relatively high temperature, can yield epitaxial single phase thin films of high crystal quality. It is therefore easier to obtain phase-pure samples with crystals oriented in a single direction and this allows for a more detailed analysis of the materials properties. One issue is that it is hardly ever possible to find a suitable substrate material with a perfectly matched lattice parameter. Lattice mismatch leads to intrinsic stress in the material and a possible distortion in the crystal lattice. Additionally, a small sample volume will render certain characterization methods impossible. When analyzing a new material with XRD, the defined orientation of the crystals will also cause problems as not all diffraction peaks are visible in one complete scan which can introduce complications with phase analysis and concurrently quantification of other phases possibly present in the deposited film. These are all issues that need to be carefully considered along with detailed chemical analysis in order to produce reliable results regarding sensitive parameters such as magnetism or transport.

In the following sections the MAX phases that have been studied experimentally in terms of magnetic characteristics are reviewed with comments, when appropriate, regarding if and how the conclusions match a coherent picture of magnetism in MAX phases.

\section{B. $\mathrm{Cr}_{2} A \mathrm{C}$ and $(\mathrm{Cr}, \mathrm{Mn})_{2} A \mathrm{C}$}

$$
\text { 1. } A=A l
$$

As stated before, $\mathrm{Cr}_{2} \mathrm{AlC}$ is the most studied MAX phase with respect to magnetism using theoretical methods. The earliest report where magnetism is discussed from experiments is by Schneider et al. [6] from 2004. They synthesized thin polycrystalline films of $\mathrm{Cr}_{2} \mathrm{AlC}$ by magnetron sputtering on cleaned and etched $\mathrm{Si}$ (111) wafers and compared the measured lattice parameters to their calculated lattice parameters for different magnetic configurations, however, no conclusions were made with respect to the specific magnetic configuration of the theoretical/experimental material. Jaouen et al. [15] studied the structure of $\mathrm{Cr}_{2} \mathrm{AlC}$ using neutron scattering on bulk samples. The samples were prepared by HIP with an excess of $10 \%$ $\mathrm{Al}$ to compensate for loss of material during the process. They found no additional peaks in the spectra (as one would expect for AFM ordering) or significant variations in the magnitude of the nuclear (Bragg) reflections, concluding, that no long range order magnetism was found in $\mathrm{Cr}_{2} \mathrm{AlC}$ in the temperature regime probed (1.8 K - $299 \mathrm{~K}$ ). Still they do report a small FM moment and assigning this to $\mathrm{Cr}$ find a considerably smaller magnetic moment $\left(\sim 0.002 \mu_{\mathrm{B}}\right)$ than has been predicted $[6,7,9,10,13]$. Their main conclusion comes from analyzing the neutron spectra at different temperatures, showing that the unit cell volume in this phase remains constant below the Curie temperature $\left(\mathrm{T}_{\mathrm{c}} \sim 73 \pm 5\right.$ 
$\mathrm{K}$ ) owing to a compensation by opposed variations (thermal/magnetic) when $\mathrm{Cr}_{2} \mathrm{AlC}$ undergoes the $\mathrm{PM}$ to FM transition. This effect is found to be similar for Invar materials which show comparable competition between thermally induced lattice variations and variations due to magnetic transitions $[105,106]$. The same group [16] later performed X-ray magnetic circular dichroism (XMCD) experiments on $\mathrm{Cr}_{2} \mathrm{AlC}$ and $\mathrm{Cr}_{2} \mathrm{GeC}$ thin films, synthesized with magnetron sputtering. The measurements were conducted at low temperatures in order to establish whether the $\mathrm{Cr}$ atoms indeed do carry a net magnetic moment. The results clearly indicate that this is the case, and XMCD recorded at the $\mathrm{Cr} L_{23}$ edges show that the $\mathrm{Cr}$ atoms carry a net magnetic moment at low temperatures for both the $A=\mathrm{Al}$ and Ge thin films. Also X-ray linear dichroism (XLD) measurements were performed and it was concluded that induced moments on both $\mathrm{Al}$ and $\mathrm{C}$ are possible although difficult to confirm experimentally.

The first experimental study on alloying $\mathrm{Cr}_{2} \mathrm{AlC}$ with Mn was performed by Mockute et al. [12], who studied $(\mathrm{Cr}, \mathrm{Mn})_{2} \mathrm{AlC}$ thin films synthesized with magnetron sputtering. Ab-initio calculations on structural properties were also included, and detailed chemical analysis of the synthesized material showed that the chemical composition was $\left(\mathrm{Cr}_{0.84} \mathrm{Mn}_{0.16}\right)_{2} \mathrm{AlC}$ and that the films were virtually single phase. These results were seen as an important step towards the $\left(\mathrm{Cr}_{0.5} \mathrm{Mn}_{0.5}\right)_{2} \mathrm{AlC}$ composition, predicted to be ferromagnetic, by Dahlqvist et al. [7]. Magnetic properties of this phase were not studied at the time, this was instead done for subsequently synthesized arc deposited films [31] found to have a composition of $\left(\mathrm{Cr}_{0.8} \mathrm{Mn}_{0.2}\right)_{2} \mathrm{AlC}$, stating this the highest $\mathrm{Mn}$ concentration in an $\mathrm{Al}$ based MAX phase to date. Magnetic characterization revealed a significant magnetic signal up to and including room temperature with virtually no change in magnetization from 5 up to $280 \mathrm{~K}$, indicating a very high transition temperature compared to other studied magnetic MAX phases. The Magn. vs field $(M / H)$ curves showed little or no hysteresis indicating soft ferromagnetism. The films were found to have a structure of discontinuous epitaxial islands of equal thickness and it was therefore difficult to determine the magnetic ordering since such a discontinuous island structure could display the observed magnetic behaviour if the material was ferromagnetic but the islands not coupled. A minor amount of $\left(\mathrm{Cr}_{1-y} \mathrm{Mn}_{y}\right)_{5} \mathrm{Al}_{8}$ with $y=0.72$ was also found in the samples by scanning (S)TEM and EDX analysis. This phase was later synthesized in pure form and studied further [107] in order to establish the possibility of it contributing to magnetic signal found for the MAX phase films. It was concluded that this could not be the case, even though the results showed that for $y=0.72$ the magnetic moment per $M$ atom in $\left(\mathrm{Cr}_{1-y} \mathrm{Mn}_{y}\right)_{5} \mathrm{Al}_{8}$ was $0.16 \mu_{\mathrm{B}}$ and consequently this number is higher then measured for the $\left(\mathrm{Cr}_{0.8} \mathrm{Mn}_{0.2}\right)_{2} \mathrm{AlC}$ sample. The study also indicated a potential issue in the synthesis of
$\left(\mathrm{Cr}_{1-x} \mathrm{Mn}_{x}\right)_{2} \mathrm{AlC}$ where the competing $\left(\mathrm{Cr}_{1-y} \mathrm{Mn}_{y}\right)_{5} \mathrm{Al}_{8}$ phase also exists as a solid solution between $\mathrm{Cr}$ and $\mathrm{Mn}$. Its crystal structure (BCC with $a=9.05 \AA$ ) was in XRD analysis also found to be virtually indistinguishable from the MAX phase deposited as an epitaxial thin film in the $[000 l]$ direction compared to the $\left(\mathrm{Cr}_{1-y} \mathrm{Mn}_{y}\right)_{5} \mathrm{Al}_{8}$ in the [ll0] direction.

Later Mockute et al. [30] attempted the bulk synthesis of both $\mathrm{Cr}_{2} \mathrm{AlC}$ and $\mathrm{Cr}_{2} \mathrm{GaC}$ with a significant addition of $\mathrm{Mn}$. A series of $(\mathrm{Cr}, \mathrm{Mn})_{2} \mathrm{AlC}$ samples with $\mathrm{Cr}: \mathrm{Mn}: \mathrm{Al}: \mathrm{C}$ molar ratios of 2:0:1.3:1, 1.8:0.2:1.3:1, 1.6:0.4:1.3:1, 1.2:0.8:1.3:1, 0.8:1.2:1.3:1, and 0.4:1.6:1.3:1 was synthesized. The powders were mixed, cold pressed and heated to $1400{ }^{\circ} \mathrm{C}$ for 1 hour under Ar flow. The sample with the Cr:Mn ratio of 1.6:0.4 was then hot pressed at $1400{ }^{\circ} \mathrm{C}$ and $39 \mathrm{MPa}$ for 3 hours. Details about the $A=$ Ga samples will be given in Section III B3. For the samples with the initial Cr:Mn ratio of 2:0, 1.8:0.2, and 1.6:0.4 the 211 MAX phase was found using powder XRD. Increasing Mn content led to the formation of the competing $\mathrm{Mn}_{3} \mathrm{AlC}$ phase with a reduction in the MAX phase. The 1.6:0.4 sample was further analysed using SEM-EDX and the results from measuring 10 different grains within the sample showed the Cr:Mn ratio to be $\sim 15: 1$ giving a final composition of $\left(\mathrm{Cr}_{0.94} \mathrm{Mn}_{0.06}\right)_{2} \mathrm{AlC}$, i.e. , $3 \% \mathrm{Mn}$ incorporation in the MAX phase structure. This composition was later confirmed using high resolution TEM and local EDX analysis of a MAX phase grain. The lattice parameters of this phase were found to be $a=2.86 \AA$ and $c=12.82$ $\AA$ which is in good agreement with $\mathrm{Cr}_{2} \mathrm{AlC}$ [68]. This is also in agreement with previous work on thin films from the same group were the addition of Mn was not found to alter the lattice parameters significantly [12], at least not beyond what would be expected due to e.g. stress in the film. For the attained composition, magnetic measurements showed no magnetic transitions in the temperature range of $2-300 \mathrm{~K}$ or any FM behavior.

$$
\text { 2. } A=G e
$$

The first magnetic MAX phase showing a ferromagnetic signal was reported by Ingason et al. [3], who studied the $\left(\mathrm{Cr}_{1-x} \mathrm{Mn}_{x}\right)_{2} \mathrm{GeC}$ phase as epitaxial thin films on $\mathrm{MgO}$ [111] substrates. Ab-initio calculations were used to establish the solubility limit of $\mathrm{Mn}$ in $\mathrm{Cr}_{2} \mathrm{GeC}$ and the results indicated that the $\left(\mathrm{Cr}_{0.75} \mathrm{Mn}_{0.25}\right)_{2} \mathrm{GeC}$ would be stable. Samples were subsequently deposited from a composite $\mathrm{Cr}_{0.75} \mathrm{Mn}_{0.25}$ target and the resulting composition was found to match the target. The calculations assume local moments on both the $\mathrm{Cr}$ and $\mathrm{Mn}$ atoms in the material, though with varying magnitudes depending on the local chemical surrounding. The calculated magnetic ground state was found to be AFM but with the FM state very close in energy. The experimental results showed a magnetic signal of the $\left(\mathrm{Cr}_{0.75} \mathrm{Mn}_{0.25}\right)_{2} \mathrm{GeC}$ thin film up to room 
temperature with a slight remanence detected up to $200 \mathrm{~K}$. The remanent magnetization, however, only accounted for one-tenth of the measured moment at $5 \mathrm{~T}$ which in turn was only a fraction of what would be expected from complete ferromagnetic ordering deduced from the calculated magnetic moments. The results therefore indicated a competition between these magnetic states, allowing for more exotic states to exist. This could then explain the observed behaviour of neither a purely AFM or FM ground state.

Liu et al. [20] studied the $\left(\mathrm{Cr}_{1-x} \mathrm{Mn}_{x}\right)_{2} \mathrm{GeC}$ phase synthesized through a solid-state reaction with $x$ up to $\sim$ 0.25. Above this ratio appreciable amounts of competing phases were found with XRD and therefore the solubility limit was estimated to $x \sim 0.25$. They characterize the magnetic behaviour of the system and suggest Mn-doped induced itinerant-electron ferromagnetism with the $\mathrm{Mn}$ pushing the parent $\mathrm{Cr}_{2} \mathrm{GeC}$ phase away from a ferromagnetic quantum critical point. The Curie temperature was found to vary with Mn concentration from 43 to $270 \mathrm{~K}$. They further claim that their data excludes any possibility of localized moments on the Mn sites in this material and that $\mathrm{Mn}$ doped $\mathrm{Cr}_{2} \mathrm{GeC}$ is actually a prototypical itinerant-electron ferromagnetic system ranging from a weak limit to a relatively well-localized regime.

Tao et al. [32] also studied $\left(\mathrm{Cr}_{1-x} \mathrm{Mn}_{x}\right)_{2} \mathrm{GeC}$ synthesized as bulk samples. They focused on exploring low concentrations of $\mathrm{Mn}$ as well as studying the phase at lower temperatures than previously done. To obtain the Mn containing samples, powders with a stoichiometry of $x=0.01,0.02,0.05$ and 0.1 were mixed, heated to 1100 ${ }^{\circ} \mathrm{C}$ and held for 48 hours. All major peaks found in the XRD diffractograms belonged to the $\left(\mathrm{Cr}_{1-x} \mathrm{Mn}_{x}\right)_{2} \mathrm{GeC}$ with only trace amounts of $\mathrm{Cr}_{5} \mathrm{Ge}_{3} \mathrm{C}_{y}$, Ge and possibly graphite. The composition of the MAX phase was hence assumed to follow the initial powder ratios. Interestingly, no significant variation of the lattice parameters with $\mathrm{Mn}$ content was found, as was observed by Liu et al. [20] for similar ratios. The magnetic behaviour was measured using a superconducting quantum interference device (SQUID) down to a temperature of $2 \mathrm{~K}$. The results had several interesting features including a possible cluster glass transitions in the sample with $x=0.01$ at $\sim 32.7$ K. Inverted hysteresis loops were also observed at $20 \mathrm{~K}$, an effect that previously has been detected in exchange coupled multilayers [108]. The authors attribute these effects to the interplay between Mn-rich regions and what they coin as the reentrant cluster matrix. They discuss a phase separation of the Mn atoms in to Mn-rich and Mn-poor regions where the Mn-rich regions exhibit higher ordering temperature which would be dependent on the Mn content [32]. For the $x=0.05$ sample, they give the saturation moment as $0.31 \mu_{\mathrm{B}}$ per $\mathrm{Mn}$ atom, which is consistent with the existence of AFM coupling.

$$
\text { 3. } A=G a
$$

The last $A$ element discussed among the synthesized MAX phases displaying magnetic properties is Ga. For synthesis in bulk, this element offers no additional challenges as compared to $\mathrm{Al}$ and $\mathrm{Ge}$, but for the production of thin films, its low melting temperature $(\sim$ $30{ }^{\circ} \mathrm{C}$ ) requires non-trivial synthesis routes.

Liu et al. [21] studied both $\mathrm{Cr}_{2} \mathrm{GaN}$ and $\mathrm{Cr}_{2} \mathrm{GaC}$ synthesized by solid-state reactions in evacuated quartz tubes. The mixture of $\mathrm{Cr}, \mathrm{C}$ and $\mathrm{Ga}$ powders was heated at $1000{ }^{\circ} \mathrm{C}$ for 24 hours while for the nitride the starting materials were $\mathrm{Cr}_{2} \mathrm{~N}$ and $\mathrm{Ga}$ and this was heated to 740 ${ }^{\circ} \mathrm{C}$ for 5 hours. In order to achieve a single phase of the materials the Ga content in the initial powder mixtures was increased through a trial and error process with the final recipe having $\sim 10 \%$ excess. The lattice parameters are claimed to agree well with those previously reported in literature $[79,109]$. The samples were measured using SQUID in the temperature range 2 - $300 \mathrm{~K}$ and resistivity measurements were performed in the same temperature range on pellets sintered from the powder. They suggest that the most probable ground state of the nitride phase is a spin-density-wave state which can be seen as a type of AFM state. On the contrary, they claim that the carbide is unambiguously non-magnetic at $4.2 \mathrm{~K}$, subsequently saying that the phase is a typical Pauli paramagnet.

Lin et al. [4] studied the $\mathrm{Cr}_{2} \mathrm{GaC}$ phase synthesized in bulk form with varying amounts of $\mathrm{Mn}$, claiming up to $50 \%$ substituting $\mathrm{Cr}$ on the $M$ sites. The samples were produced by the direct reaction of the constituent elements where the materials were mixed in desired proportions, sealed in evacuated quartz tubes and then annealed at $850-900{ }^{\circ} \mathrm{C}$ for five days. The samples were subsequently pulverized, pressed into pellets and further annealed at $1000{ }^{\circ} \mathrm{C}$ for eight days to ensure homogeneity. For samples containing a high concentration of $\mathrm{Mn}(x=0.75-1.00)$, a significant amount of the competing $\mathrm{Mn}_{3} \mathrm{GaC}$ phase as well as $\mathrm{C}$ was found. Below this Mn value, no significant peaks were found with XRD belonging to other phases than the $\left(\mathrm{Cr}_{1-x} \mathrm{Mn}_{x}\right)_{2} \mathrm{GaC}$ phase. The sample composition was therefore assumed to be the same as for the virgin powder concentration. The authors observe a decreasing unit cell volume with Mn content, along with a decrease in thermal conductivity and an increase in electrical resistivity. They also claim that the introduction of Mn changes the magnetism in the material from a non-magnetic $\mathrm{Cr}_{2} \mathrm{GaC}(x=0)$ to ferrimagnetic ordering for the $x=0.5$ composition. This is corroborated by a tiny anomaly in the $M / H$ curve at $5 \mathrm{~K}$ at a low magnetic field.

Guided by predictions from ab-initio calculations, Ingason et al. [25] advanced beyond alloying and synthesized $\mathrm{Mn}_{2} \mathrm{GaC}$ samples as a heteroepitaxial thin film on $\mathrm{MgO}[111]$ substrates. The samples were co-sputtered from C, Mn and Ga targets. Due to the low melting temperature of gallium $\left(\sim 30^{\circ}\right)$ the sputtering 
target melts during the sputtering process and must therefore be kept in a stainless steel crucible. The details about the experimental setup can be found in [22, 110]. The $\mathrm{Mn}_{2} \mathrm{GaC}$ sample was virtually phase pure of high structural quality with minor traces of the $\mathrm{Mn}_{3} \mathrm{GaC}$ phase. Being the sole $M$ element, $M n$ was for the first time introduced as a new member of the MAX phases family, potentially expanding the number of possible MAX phases. This also showed that the solid solution between $\mathrm{Cr}$ and $\mathrm{Mn}$ on the $M$ site in $M_{2} \mathrm{GaC}$ could likely exist at any ratio, since the end members both exist, and that the issue of obtaining phase pure samples with high Mn content is not a matter of inherent thermal stability of that phase, but rather the fine tuning of the experimental procedure.

$\mathrm{Mn}_{2} \mathrm{GaC}$ was found to display a FM signal at temperatures below $230 \mathrm{~K}$ with the remanent magnetization, $m_{r}=0.13 \mu_{\mathrm{B}}$ per $\mathrm{Mn}$ atom. The predicted moment per Mn atom for the FM configuration is, according to calculations, close to $1.6 \mu_{\mathrm{B}}$. Hence, the ferromagnetic signal detected can not originate from fully ferromagnetic ordering in the material, and more complicated configurations need to be considered.

Going further with measurements of the magnetic properties of $\mathrm{Mn}_{2} \mathrm{GaC}$, Dahlqvist et al. [27] performed vibrating sample magnetromety (VSM), XRD and magneto-optic Kerr effect (MOKE) measurements at low temperatures. In conjunction, more detailed ab-initio calculations were performed to gain further insight. The results showed that this system has a rich magnetic phase diagram with several magnetic configurations almost equivalent in energy. Which of these configurations the system occupies was shown to depend on the out-of-plane lattice parameter and hence on the temperature. The authors showed that the system undergoes temperature dependent transitions between magnetic configurations that coincide with drastic changes in the out-of-plane lattice parameter. Further, some of the theoretical spin configurations found will induce new symmetries in the crystal structure of the MAX phase, giving rise to new diffraction peaks, which was also experimentally observed. These structural changes are inherently anisotropic since they occur due to the interplay between the magnetic configurations and the layered structure, driven by the magnetic configuration, rather than vise versa. The three lowest energy configurations were the FM and two different AFM configurations denoted $\operatorname{AFM}[0001]_{2}^{A}$ and $\operatorname{AFM}[0001]_{4}^{A}$, where two or four consecutive Mn layers are found to be co-parallel with the direction of the spins flipping across an $A$ (Ga) layer, see Fig. 2. The energy of those configurations were the spins are flipped across the $X(\mathrm{C})$ layer was consistently found to be higher. It was therefore concluded that the $\mathrm{Mn}$ in Mn-C-Mn layers are always spin-parallel and constitute the magnetic unit of the material. The material does show ferromagnetic behavior below $240 \mathrm{~K}$, and at the lowest temperature measured, $2.4 \mathrm{~K}$ and highest field applied, $5 \mathrm{~T}$, the magnetic signal measured corresponded to $1.6 \mu_{\mathrm{B}}$ per $\mathrm{Mn}$ atom. The AFM configuration the system occupies is therefore seen as non-collinear with a certain canting angle that can be induced with very low cost in energy, giving rise to the ferromagnetic component. Subsequent increase in the applied field pushes the spins further until at $5 \mathrm{~T}$ the system is very close to being collinear.

To gain more insight into the magnetism in $\mathrm{Mn}_{2} \mathrm{GaC}$, Ingason et al. [29] performed neutron reflectivity measurements on a $50 \mathrm{~nm}$ thin $\mathrm{Mn}_{2} \mathrm{GaC}$ sample. They showed unambiguously that the material has long range magnetic order, exemplified by clear neutron diffraction peaks (see Fig. 5) that could only occur due to magnetic repetition. The peaks observed corresponded to that of a magnetic repetition distance of two unit cells which complies with the proposed AFM $[0001]_{4}^{A}$ from Dahlqvist et al. [27] but also a host of other configurations with non-collinear ordering.

Following the work on $\mathrm{Mn}_{2} \mathrm{GaC}$, thin epitaxial films of the alloy $\left(\mathrm{Cr}_{0.5} \mathrm{Mn}_{0.5}\right)_{2} \mathrm{GaC}$ were synthesized on various substrates at $\sim 550{ }^{\circ} \mathrm{C}$ by Petruhins et al. [33]. The films were co-sputtered from $\mathrm{Ga}, \mathrm{C}$ and a composite $\mathrm{Cr}_{0.5} \mathrm{Mn}_{0.5}$ targets. The crystal quality of the films, in particular on $\mathrm{MgO}$ substrates, was very high with the best results to date obtained by XRD, evident from rocking curve measurements performed on the MAX phase 0006 peak. This was confirmed through high resolution TEM (HRTEM) measurements and a sample composition of $\left(\mathrm{Cr}_{0.5} \mathrm{Mn}_{0.5}\right)_{2} \mathrm{GaC}$ was confirmed using EDX. Magnetic measurements showed a significant response throughout the temperature regime tested $(30-300 \mathrm{~K})$ with the highest measured moment at $30 \mathrm{~K}$ and $5 \mathrm{~T}$ being $0.67 \mu_{\mathrm{B}}$ per $M$ atom. Slight remanence was found as well with the highest remanent moment of $0.036 \mu_{\mathrm{B}}$ per $\mathrm{Mn}$ atom. The temperature dependence of the magnetization did not follow typical Curie-Weiss behaviour, rather, the slow transition of the behaviour at low temperature $(30 \mathrm{~K})$ to room temperature was characterized as a competition between FM and AFM states or what could be described as a non-collinear AFM state. Salikhov et al. [34] later characterized the same films using ferromagnetic resonance at temperatures between 110 and $300 \mathrm{~K}$. Their results reveal a ferromagnetic $g$-factor of $2.00 \pm 0.01$ over the entire temperature range, suggesting a small orbital contribution to the magnetic moment. The magnetic moments were found to lie in the basal plane of the phase and they consequently classify this material as a quasi $2 \mathrm{D}$ laminar ferromagnet.

\section{Other magnetic MAX phases}

The high stability of $\mathrm{Mn}_{2} \mathrm{GaC}$ motivates alloying this phase with others elements, beyond $\mathrm{Cr}$, to study the effect of composition on the magnetic properties. 


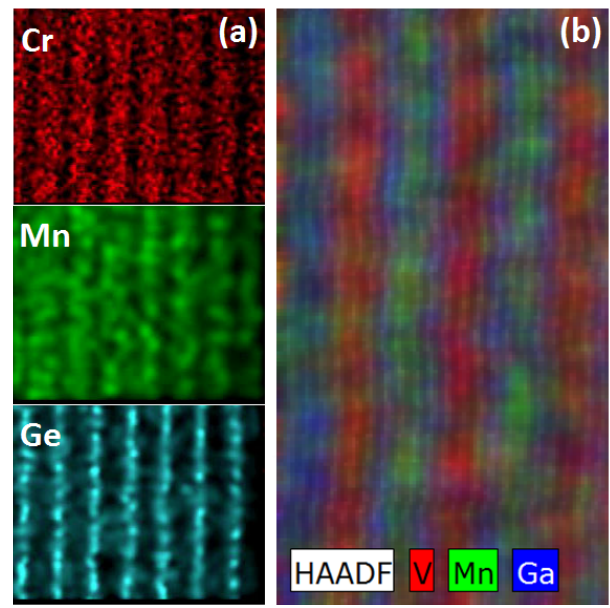

Figure 3. Example of (a) $M$ element disorder in $(\mathrm{Cr}, \mathrm{Mn})_{2} \mathrm{GeC}$, and (b) $M$ element order in $(\mathrm{V}, \mathrm{Mn})_{3} \mathrm{GaC}_{2}$, from electron microscopy.

\section{1. $\left(\mathrm{Mo}_{0.5} \mathrm{Mn}_{0.5}\right)_{2} \mathrm{GaC}$}

Meshkian et al. [35] synthesised $\left(\mathrm{Mo}_{0.5} \mathrm{Mn}_{0.5}\right)_{2} \mathrm{GaC}$ as heteroepitaxial thin films on $\mathrm{MgO}$ [111] substrates. The samples were co-sputtered from a $\mathrm{Ga}$ and $\mathrm{C}$ target as well as a composite $\mathrm{Mo}_{0.5} \mathrm{Mn}_{0.5}$ target. The $M$ element composition measured with TEM-EDX was found to match the target composition, and the crystal quality was found to be high and the overall composition uniform. The material displayed a clear FM response up to and including $150 \mathrm{~K}$, with a measured moment at $5 \mathrm{~T}$ and a remanent moment (both at $3 \mathrm{~K}$ ) of 0.66 and $0.35 \mu_{\mathrm{B}}$ per $M$ atom, respectively. This value for the remanent moment and the measured value for the coercive field $(0.06 \mathrm{~T})$ are the highest reported to date for magnetic MAX phases.

\section{2. $(\mathrm{V}, \mathrm{Mn})_{3} \mathrm{GaC}_{2}$}

Until most recently, all MAX phases claimed to be magnetic were confined to a 211 stoichiometry. However, using $\mathrm{Mn}_{0.5} \mathrm{~V}_{0.5}, \mathrm{Ga}$, and $\mathrm{C}$ targets, Tao et al. [36] synthesized a $(\mathrm{V}, \mathrm{Mn})_{3} \mathrm{GaC}_{2} \mathrm{MAX}$ phase as an epitaxial thin film on a $\mathrm{MgO}[111]$ substrates. From SQUID measurements, a FM response well above $350 \mathrm{~K}$ could be concluded, with a remanent moment at $100 \mathrm{~K}$ of approximately $0.04 \mu_{\mathrm{B}}$ per $M$ atom. As opposed to all previous magnetic MAX phase alloys showing $M$ element disorder, exemplified in Figure 3(a), high resolution (S)TEM-EDX showed evidence of chemical ordering with separation of V and Mn in separate layers, see Fig. 3(b). These initial results are promising for future prospects of magnetic MAX phase (super-) structures.

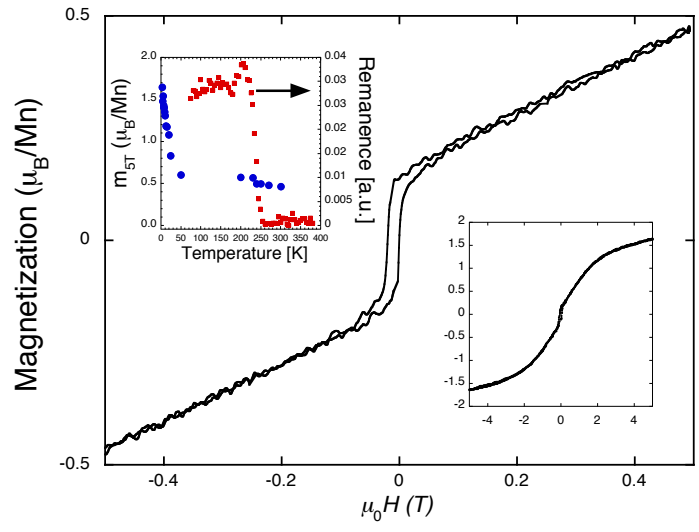

Figure 4. Typical magnetization vs. field behaviour of a MAX phase, shown here for a thin film $\mathrm{Mn}_{2} \mathrm{GaC}$ sample [27] at 3 $\mathrm{K}$. The inset on the lower right shows the magnetization up to $5 \mathrm{~T}$ while the inset on the left shows the magnetization at $5 \mathrm{~T}\left(\mathrm{~m}_{5 T}\right)$ as well as the remancence behavior as functions of temperature.

\section{MAGNETIC CHARACTERISTICS}

Due to the many different material compositions being studied, as well as variations within those systems and issues with different synthesis routes and thereby differences in sample type, quality etc., it is not straightforward to get more than a superficial view of the general magnetic characteristics of these materials. This evident from the discrepancies found in tables II and III with respect to calculated vs. measured magnetic moments where measured magnetic moments are sometimes one or two orders of magnitude lower than calculated ones. The exact reasons for this discrepancy are unknown but we can assume that they relate to sample quality, uncertainty about the exact nature of the magnetism and therefore how data is interpreted as well as to the approximations and methods used. These issues are discussed in more detail below. Overall the materials seem to display weak ferromagnetism at relatively low temperatures with some materials indicating transition temperatures above room temperature. Several phases also show increased magnetization with field beyond the remanent field. This is exemplified in the typical magnetization vs. field $(M / H)$ curve shown in Fig. 4 for a thin $\mathrm{Mn}_{2} \mathrm{GaC}$ film at $4 \mathrm{~K}$ (data from [27]). The right inset shows the same data for higher applied field. The inset on the left displays the magnetization at $5 \mathrm{~T}\left(\mathrm{~m}_{5 T}\right)$ as well as the remanence behavior as function of temperature. Several different views have been published regarding the origin of the magnetism in MAX phases, with the aforementioned issues complicating the work of consolidating those views. The magnetic behaviour has been suggested to originate from itinerant-electron ferromagnetism, spin-density-wave states, Pauli paramagnetism, non-collinear local spin ordering etc. The great interest in these materials 
coupled with the exciting prospect for their application will surely mean that this will be solved in the near future.

\section{A. Effect of sample quality on magnetic properties}

The issue of sample quality and reproducibility has prevalence in all areas of materials physics. This is especially apparent for the relatively new research field as magnetism in MAX phases. When discussing sample quality there is a need to define what is meant by quality and the degree of quality that is needed in order to draw conclusions from measurements performed on those samples. First to mention is phase purity, i.e., the sample being measured is composed solely of the phase being studied. The second issue is the detailed composition of that phase with respect to the alloying elements, i.e., what is the amount of e.g. Mn that actually is incorporated into the MAX phase structure, substituting another $M$ element. Thirdly, once the detailed composition of the sample has been established, its inherent crystal quality can be discussed as an optimization parameter, which becomes more important e.g. once magnetic measurements become more detailed.

Most of the systems that have been studied are ternary systems alloyed with the fourth element meaning that the number of stable competing phases possibly existing in the vicinity of the composition being studied increases considerably. Additionally, those competing phases are often closely related to the MAX phase in question, and so, if a solid solution is expected to exist between those elements in the MAX phase, the same goes for the competing phase. That is to say, if traces of a competing phase which according to literature only contains non-magnetic elements are found, chances are that the magnetic element has also been alloyed into that phase, thus excluding it from the analysis might give false conclusions.

\section{B. Effect of $M, A$ and $X$ elements on magnetic properties}

So far the sample quality and the detail of measurements have not come to a point where we can say for sure what are the differences between magnetic MAX phases when changing from one $M, A$ or $X$ element to another. Attempts have been made to answer this question but given the different conditions needed and different set of competing phases involved, few studies have succeeded in synthesizing samples that only differ by one element allowing for the magnetic properties to be reliably compared.

Regarding the $M$ element is is known that increasing the Mn content in a phase composed of Mn and e.g., Cr, will give a more pronounced magnetic signal. Also, the local ordering between $\mathrm{Mn}$ and $\mathrm{Cr}$ will very likely have a critical impact on the magnetism observed, as indicated by previous theoretical work [7].

For the $A$ elements, what is clearly known is that among the most studied elements, Ga is the only one were a phase with a sole $M$ element that displays a ferromagnetic response can be obtained $\left(\mathrm{Mn}_{2} \mathrm{GaC}\right)$. That is, solubility ratio between e.g. $\mathrm{Cr}$ and $\mathrm{Mn}$ depends heavily on the $A$ element. Also known is that the highest observed transition temperature is observed for $A=\mathrm{Al}$ in [31].

For the $X$ element, Liu et al. [21] do synthesize $\mathrm{Cr}_{2} \mathrm{GaC}$ and $\mathrm{Cr}_{2} \mathrm{GaN}$ thereby allowing a comparison of the effect of the $X$ element, but since these materials are either strongly AFM, PM or NM there is little or no magnetic response to compare. The main difference observed is in the temperature dependence of the resistivity, where there is a kink in the data for the nitride, characteristic of a spin-density-wave state, also observed for pure Cr [111, 112]. Generally one would expect a drastic difference between a magnetic MAX phase carbide and the corresponding nitride, since in similar systems the nitride phase will typically give rise to higher transition temperatures. This, for reasons discussed below, has not been much explored.

\section{Local vs. itinerant magnetism}

The question of itinerant vs. local magnetism in these systems was first raised in a publication by Liu et al. [20]. The authors analyze data obtained from their bulk samples of $\mathrm{Mn}$ doped $\mathrm{Cr}_{2} \mathrm{GeC}$ and obtain values for the Curie temperature $T_{\mathrm{C}}$, the effective magnetic moment, $\rho_{\text {eff }}$, and the spontaneous magnetic moment $\rho_{\mathrm{s}}$ as a function of the Mn content up to $x=0.25$ in $\left(\mathrm{Cr}_{1-x} \mathrm{Mn}_{x}\right)_{2} \mathrm{GeC}$. The results show that at low $\mathrm{Mn}$ content the ratio $\rho_{\text {eff }} / \rho_{\mathrm{s}}$ is much larger than unity, meaning that the system is in the regime of a very weak itinerant-electron ferromagnet [20]. As the Mn content increases $\rho_{\text {eff }} / \rho_{\mathrm{s}}$ decreases rapidly, approaching the local moment limit. The authors thereafter conclude that a local moment on the Mn sites can be excluded.

For the example above, it is assumed that in the regime where a ferromagnetic signal is detected the sample is fully saturated and therefore allows use of the conventional Rhodes-Wolfarth [113] plot to establish the relationship between values of $\rho_{\text {eff }} / \rho_{\mathrm{s}}$ against $T_{\mathrm{C}} / T_{0}$, routinely used to identify itinerant systems. As we have seen numerous times, these materials often show remanence from conventional $M / H$ plots while the magnetization is seen to increase beyond the remanent magnetization, meaning that material cannot be assumed to be saturated at the cohesive field. Wohlfarth [114] expresses a word of caution when the material being treated has unusual spin structures below the Curie point, such as ferrimagnetic, conical, or spiral, since $\rho_{\text {eff }} / \rho_{\mathrm{s}}$ may then also be larger than unity and the method inconclusive. 
There is still the overall consensus that even though not all cases are fully understood at present, the majority of metallic magnets, excluding only rare-earth magnetic materials, can be explained within the itinerant electronic picture. The electrons contributing to magnetism also participate in the Fermi surface and can therefore all be characterized as being itinerant. They do however not necessarily form delocalized magnetic moments, but conspire to such an extent that the moments are localized on some scale [115]. This essentially means that we are not dealing with two distinct cases, local or itinerant, but with a distribution which requires a unified treatment that interpolates between the two [115]. The Wolfarth-Rhodes ratio has been reported for e.g., Fe, Co and $\mathrm{Ni}$ to be 1.03, 1.34 and 1.46 respectively, while for $\mathrm{MnSi}$, considered a weak itinerant ferromagnet, the ratio is found to be 3.5 [116]. The important point is establishing whether the theoretical methods used hitherto to describe these materials are fully applicable or if they need to be complemented with methods that deal more appropriately with highly correlated systems.

\section{Collinear vs. non-collinear spin configurations}

The apparent discrepancy between the observed ferromagnetic behaviour of some magnetic MAX phases coupled with an increase in the magnetic response with field beyond the remanent field, has been explained in terms of non-collinear magnetic structures. Given that the materials display ferromagnetism or a ferromagnetic component, they cannot be characterized as pure antiferromagnets. Furthermore, the low remanent magnetization they display, compared to what could be expected from a fully collinear ferromagnet, shows that they are not ferromagnets. Non-collinearity was first introduced by Ingason et al. [25] and further elaborated in Ref. [27].

The explanation broadly states that the system can occupy multitude of different low energy spin configurations, some of which are non-collinear, and give a net magnetic moment. These non-collinear configuration can be referred to as canted-AFM structures and their measured behavior is similar to ferromagnetism but with a much reduced magnetization as compared to the FM configuration. The ferromagnetic configuration is however close in terms of energy, so as the field is increased beyond the remanent field, the spins are rotated further towards it, eventually becoming collinear. The most recent results from neutron diffraction of $\mathrm{Mn}_{2} \mathrm{GaC}$ confirm the existence of an AFM state for this phase [29] and lends credence to the suggested non-collinearity, but further work is still required.

\section{OUTLOOK}

\section{A. Tuning of properties}

Even though the community has just started to thoroughly establish the magnetic ground state and the magnetic behaviour in relevant MAX phases, one can already begin to see the possibilities that these materials may offer in terms of tunable properties. For any given application, the relevant parameters such as transition temperatures, type of magnetization etc. likely need to be optimized. This can only be achieved if the material lends itself to tuning, i.e. that by changing selected internal parameters of the material in some way, without affecting its crystal structure, its magnetic properties can be altered.

First, obviously, the chemical ratio between two $M$ elements can be varied. As an example, the existence of the ternary $\mathrm{Mn}_{2} \mathrm{GaC}$ and $\mathrm{Cr}_{2} \mathrm{GaC}$ as well as the corresponding alloy $\left(\mathrm{Cr}_{0.5} \mathrm{Mn}_{0.5}\right)_{2} \mathrm{GaC}$ indicates that a sample at any ratio between the two $M$ metals can likely be synthesized, opening a route for tuning. Further, the chemical ordering of those alloys can be changed by e.g., creating whole layers of different thicknesses of each metal carbide while keeping the atomic ratio between them fixed. Calculations have indicated that this also would be a route for tuning their magnetic properties. Alloying on the $A$-site is another possible route [117], motivated by the difference between the magnetic behaviour of those MAX phases with different $A$ elements studied to date. Turning to the $X$ elements, there is only one experimental study [21] which has dealt with a nitride counterpart to the more traditional carbide, however, alloying between carbon and nitrogen will most likely also alter the magnetic behavior. Furthermore, defects such as vacancies is an alternate path for tuning, motivated by reports [118] indicating a significant change in the related carbide properties due to variations in their stoichiometry. A great benefit of MAX phases is that the material will retain its structure through all suggested compositional variations and that different MAX phases that have been tuned in different ways can be grown together in a stack to maximize the desired effect. This is the case for various properties that might be of interest, be it optical, mechanical, calorimetric or indeed magnetic.

\section{B. Techniques for further evaluations of magnetism}

Out of the currently published papers that deal with magnetism in MAX phases only a few deviate from the traditional VSM/SQUID bulk measurement techniques. Moving forward, there is a need to expand the range of techniques used to map out the magnetic ground states and behavior of these materials. For example, only two publications have come out where neutrons are used to gain insight into the magnetic structure 


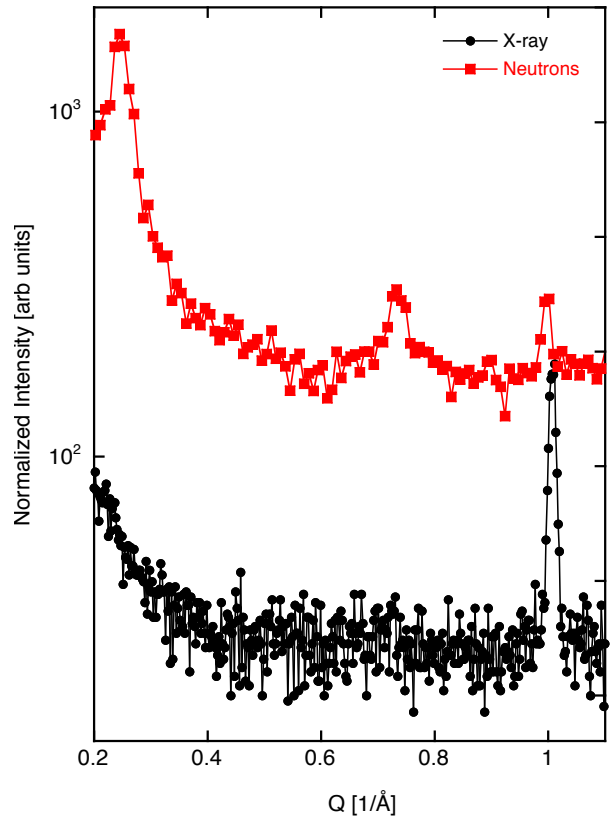

Figure 5. Neutron scattering (square icons) of an epitaxial $\mathrm{Mn}_{2} \mathrm{GaC}$ thin film at $150 \mathrm{~K}$ compared to XRD (circles) at the same temperature. The peaks at $Q=0.25$ and 0.75 , not present in the X-ray data, occur due to magnetic repetition in the material and therefore show unequivocally that this material has long range magnetic order. The period is consistent with a magnetic repetition distance of two unit cells in the $c$ direction [29].

of a MAX phase material [29] and [15]. Due to the considerable time these measurements take, theoretical simulations can be used prior to data acquisition to suggest the modulation distance chosen for probing. This decreases the risk of time being spent measuring ranges that are not of interest or relevant ranges being ignored completely. As an example Figure 5 shows neutron scattering for an epitaxial $\mathrm{Mn}_{2} \mathrm{GaC}$ thin film at $150 \mathrm{~K}$. The scan is performed at very low $\mathrm{Q}$ values, well below the first allowed structural peak $(0002)$ at $Q \approx 1$ due to the large modulation distances proposed by theory [27]. Without the theoretical prediction, this range might have been ignored, and the clear peaks at $Q=0.25$ and 0.75 proving long range magnetic order in this phase missed. In addition to this, chemically sensitive methods such as XMCD or electron magnetic circular dichroism (EMCD) have only been use in a limited number of publications but could give valuable insight into the magnetic behavior of these phases, unobtainable by other methods.

\section{Additional research routes for magnetic nanolaminates}

In addition to investigations on the interplay between sample parameters, quality, and magnetic behavior, there

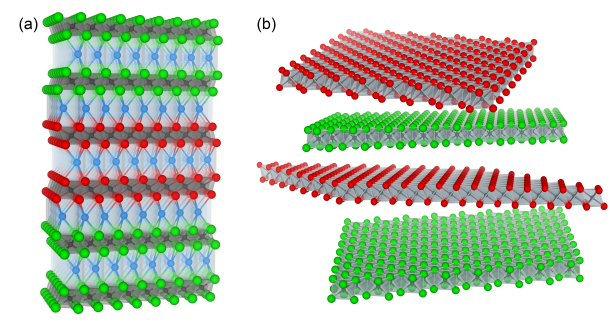

Figure 6. (a) Different MAX phases grown in a single crystal stack. (b) MXene from selective etching of the $A$ layer.

are a multitude of phases that have the same basic structural properties as MAX phases that remain to be explored. Early on in this field of research there were attempts of experimentally verifying theoretical predictions [7] which indicated that in the case of the $(\mathrm{Cr}, \mathrm{Mn})_{2} \mathrm{AlC}$ phases, the ordered stacking, i.e., whole layers of $\mathrm{Mn}$ and $\mathrm{Cr}$, as opposed to random mixing, would be energetically more favourable. This has to date not been experimentally shown, and no results indicate that samples that are synthesized with random chemical ordering can migrate towards order. Synthesizing these ordered phases would likely show a behaviour contrasting with what has so far been observed. The first material in this direction is the magnetic $(\mathrm{V}, \mathrm{Mn})_{3} \mathrm{GaC}_{2}$, mentioned above, which displays chemical order and with a driving force for separating $\mathrm{V}$ and $\mathrm{Mn}$.

Related to this is the possibility of growing stacks of different MAX phases, see Fig. 6(a). This will likely be realized given the fact that the lattice parameters $a$ and $b$ of MAX phases differ only slightly and that they are routinely deposited on substrates with different in-plane lattice parameters than themselves. Hence, they can be used as building blocks for devices where each layer serves a given purpose and together compose a single crystal stack throughout.

In addition to the above, the past couple of years has seen a whole host of discoveries of new materials that relate to the MAX phases. These have a slightly different ordering and stoichiometry compared to traditional MAX phases but share the hexagonal layered structure. Examples are $\left(\mathrm{Cr}_{2 / 3} \mathrm{Ti}_{1 / 3}\right)_{3} \mathrm{AlC}_{2}$ and $\left(\mathrm{Cr}_{5 / 8} \mathrm{Ti}_{3 / 8}\right)_{4} \mathrm{AlC}_{3}$ [40], as well as the recently discovered $\mathrm{Mo}_{2} \mathrm{Ga}_{2} \mathrm{C}[87,119]$ which is essentially the same phase as the 211 MAX phase but with two subsequent Ga layers. Another example is $\left(\mathrm{Cr}_{0.5} \mathrm{~V}_{0.5}\right)_{n+1} \mathrm{AlC}_{n}$ [83] which belongs to a group of materials which can be characterized as being composed of two different MAX phases [39]. The multitude of new phases sharing some of the attributes that make magnetic MAX phases an intriguing research subject indicates, that we are just starting to scratch the surface in this field. There is likely to be a great number of so far undiscovered phases, containing elements that would endow them with novel magnetic properties.

Two-dimensional (2D) materials are known to 
have unique properties typically differing from their corresponding 3D structures, and another future research route is the quest of a magnetic $2 \mathrm{D}$ counterpart to the MAX phases, i.e., magnetic MXene, see Fig. 6(b). Typically, the $A$ element is etched from the structure leaving only the $M_{n+1} \mathrm{C}_{n}$ layers $[120,121]$. However, to date, no magnetic MAX phases have been successfully etched to magnetic MXene. Another aspect of magnetic MAX phases that remains to a large extent unexplored is properties beyond magnetism. A few such examples are transport behavior, calorimetric properties (given the intriguing properties of e.g. the closely related inverse perovskites), and whether or not these phases can be used for spin-transport.

Finally, we stress the importance of systematic experimental studies, preferably in conjunction with predictive and explanatory theoretical work, to elucidate the role of chemistry and structure on the fundamental magnetic properties. This will show to which extent these materials can be engineered and the properties tuned, for identification of novel MAX phases with interesting and attractive properties.

\section{ACKNOWLEDGMENTS}

We acknowledge support from European Research Council (ERC), the Swedish Research Council (VR), grant no. 621-2012-4425 and 642-2013-8020, the KAW foundation, and the Swedish Foundation for Strategic Research (SSF) through the synergy grant FUNCASE.
[1] V. H. Nowotny, Progress in Solid State Chemistry 5, 27 (1971).

[2] M. W. Barsoum, MAX Phases, Properties of Machinable Ternary Carbides and Nitrides (Wiley-VCH Verlag GmbH \& Co. KGaA, Weinheim, Germany, 2013).

[3] A. S. Ingason, A. Mockute, M. Dahlqvist, F. Magnus, S. Olafsson, U. B. Arnalds, B. Alling, I. A. Abrikosov, B. Hjörvarsson, P. O. Å. Persson, and J. Rosén, Physical Review Letters 110, 195502 (2013).

[4] S. Lin, P. Tong, B. S. Wang, Y. N. Huang, W. J. Lu, D. F. Shao, B. C. Zhao, W. H. Song, and Y. P. Sun, Journal of Applied Physics 113, 053502 (2013).

[5] Z. Sun, R. Ahuja, S. Li, and J. M. Schneider, Applied Physics Letters 83, 899 (2003).

[6] J. M. Schneider, Z. Sun, R. Mertens, F. Uestel, and R. Ahuja, Solid State Communications 130, 445 (2004).

[7] M. Dahlqvist, B. Alling, I. A. Abrikosov, and J. Rosén, Physical Review B 84, 220403 (2011).

[8] Y. L. Du, Z. M. Sun, H. Hashimoto, and M. W. Barsoum, Journal of Applied Physics 109, 063707 (2011).

[9] M. Ramzan, S. Lebègue, and R. Ahuja, physica status solidi (RRL) - Rapid Research Letters 5, 122 (2011).

[10] M. Dahlquist, B. Alling, and J. Rosén, Journal of Applied Physics 113, 216103 (2013).

[11] W. Sun, W. Luo, and R. Ahuja, Journal of Materials Science 47, 7615 (2012).

[12] A. Mockute, M. Dahlqvist, J. Emmerlich, L. Hultman, J. M. Schneider, P. O. Å. Persson, and J. Rosén, Physical Review B 87, 094113 (2013).

[13] M. Dahlqvist, B. Alling, and J. Rosén, Journal of Physics: Condensed Matter 27, 095601 (2015).

[14] M. Dahlqvist, B. Alling, and J. Rosén, Physical Review B 81, 220102(R) (2010).

[15] M. Jaouen, P. Chartier, T. Cabioch, V. Mauchamp, G. André, and M. Viret, Journal of the American Ceramic Society 96, 3872 (2013).

[16] M. Jaouen, M. Bugnet, N. Jaouen, P. Ohresser, V. Mauchamp, T. Cabioch, and A. Rogalev, Journal of Physics: Condensed Matter 26, 176002 (2014).

[17] M. Ramzan, S. Lebègue, and R. Ahuja, Solid State Communications 152, 1147 (2012).
[18] N. Li, C. C. Dharmawardhana, K. L. Yao, and W. Y. Ching, Solid State Communications 174, 43 (2013).

[19] M. Mattesini and M. Magnuson, Journal of Physics: Condensed Matter 25, 035601 (2013).

[20] Z. Liu, T. Waki, Y. Tabata, and H. Nakamura, Physical Review B 89, 054435 (2014).

[21] Z. Liu, T. Waki, Y. Tabata, K. Yuge, H. Nakamura, and I. Watanabe, Physical Review B 88, 134401 (2013).

[22] A. Petruhins, A. S. Ingason, M. Dahlqvist, A. Mockute, M. Junaid, J. Birch, J. Lu, L. Hultman, P. O. A. Persson, and J. Rosén, physica status solidi (RRL) Rapid Research Letters 7, 971 (2013).

[23] M. Benouis, Y. Azzaz, M. Ameri, O. Arbouche, A. Bennadji, D. Bensaid, and Y. Al-Douri, Journal of Superconductivity and Novel Magnetism 29, 1267 (2016).

[24] W. Luo and R. Ahuja, Journal of Physics: Condensed Matter 20, 064217 (2008).

[25] A. S. Ingason, A. Petruhins, M. Dahlqvist, F. Magnus, A. Mockute, B. Alling, L. Hultman, I. A. Abrikosov, P. O. A. Persson, and J. Rosén, Materials Research Letters 2, 89 (2013).

[26] A. Thore, M. Dahlqvist, B. Alling, and J. Rosén, Journal of Applied Physics 116, 103511 (2014).

[27] M. Dahlqvist, A. S. Ingason, B. Alling, F. Magnus, A. Thore, A. Petruhins, A. Mockute, U. B. Arnalds, M. Sahlberg, B. Hjörvarsson, I. A. Abrikosov, and J. Rosén, Physical Review B 93, 014410 (2016).

[28] A. Thore, M. Dahlqvist, B. Alling, and J. Rosén, Physical Review B 93, 054432 (2016).

[29] A. S. Ingason, G. K. Pálsson, M. Dahlqvist, and J. Rosén, Physical Review B.

[30] A. Mockute, J. Lu, E. J. Moon, M. Yan, B. Anasori, S. J. May, M. W. Barsoum, and J. Rosén, Materials Research Letters 3, 16 (2014).

[31] A. Mockute, P. O. Å. Persson, F. Magnus, A. S. Ingason, S. Olafsson, L. Hultman, and J. Rosén, physica status solidi (RRL) - Rapid Research Letters 8, 420 (2014).

[32] Q. Z. Tao, C. F. Hu, S. Lin, H. B. Zhang, F. Z. Li, D. Qu, M. L. Wu, Y. P. Sun, Y. Sakka, and M. W. Barsoum, Materials Research Letters 2, 192 (2014).

[33] A. Petruhins, A. S. Ingason, J. Lu, F. Magnus, 
S. Olafsson, and J. Rosén, Journal of Materials Science 50, 4495 (2015).

[34] R. Salikhov, A. S. Semisalova, A. Petruhins, A. S. Ingason, J. Rosén, U. Wiedwald, and M. Farle, Materials Research Letters 3, 156 (2015).

[35] R. Meshkian, A. S. Ingason, U. B. Arnalds, F. Magnus, J. Lu, and J. Rosén, APL Materials 3, 076102 (2015).

[36] Q. Tao, R. Salikhov, A. Mockute, J. Lu, M. Farle, U. Wiedwald, and J. Rosén, APL Materials 4, 086109 (2016).

[37] C. Li, Z. Wang, D. Ma, C. Wang, and B. Wang, Intermetallics 43, 71 (2013).

[38] Z. Liu, E. Wu, J. Wang, Y. Qian, H. Xiang, X. Li, Q. Jin, G. Sun, X. Chen, J. Wang, and M. Li, Acta Materialia 73, 186 (2014).

[39] M. Dahlqvist and J. Rosén, Physical Chemistry Chemical Physics 17, 31810 (2015).

[40] Z. Liu, L. Zheng, L. Sun, Y. Qian, J. Wang, and M. Li, Journal of the American Ceramic Society 97, 67 (2013).

[41] D. Horlait, S. Grasso, N. Al Nasiri, P. A. Burr, and W. E. Lee, Journal of the American Ceramic Society 99, $682(2015)$.

[42] P. Hohenberg and W. Kohn, Physical Review 136, B864 (1964).

[43] W. Kohn and L. J. Sham, Physical Review 140, A1133 (1965).

[44] R. M. Martin, "Electronic structure: basic theory and practical methods," Cambridge University Press, Cambridge (2004).

[45] A. D. Becke, Physical Review A 38, 3098 (1988).

[46] J. P. Perdew, K. Burke, and M. Ernzerhof, Physical Review Letters 77, 3865 (1996).

[47] J. P. Perdew, J. A. Chevary, S. H. Vosko, K. A. Jackson, M. R. Pederson, D. J. Singh, and C. Fiolhais, Physical Review B 46, 6671 (1992).

[48] L. Wang, T. Maxisch, and G. Ceder, Physical Review B 73, 195107 (2006).

[49] G. Hautier, S. P. Ong, A. Jain, C. J. Moore, and G. Ceder, Physical Review B 85, 155208 (2012).

[50] M. Aykol and C. Wolverton, Physical Review B 90, 115105 (2014).

[51] B. Alling, T. Marten, and I. A. Abrikosov, Physical Review B 82, 184430 (2010).

[52] A. S. Botana, F. Tran, V. Pardo, D. Baldomir, and P. Blaha, Physical Review B 85, 235118 (2012).

[53] B. Alling, A. Khatibi, S. I. Simak, P. Eklund, and L. Hultman, Journal of Vacuum Science \& Technology A: Vacuum, Surfaces, and Films 31, 030602 (2013).

[54] N. J. Mosey and E. A. Carter, Physical Review B 76, 155123 (2007).

[55] A. I. Liechtenstein, V. I. Anisimov, and J. Zaanen, Physical Review B 52, R5467 (1995).

[56] S. L. Dudarev, G. A. Botton, S. Y. Savrasov, and C. J. Humphreys, Physical Review B 57, 1505 (1998).

[57] A. G. Petukhov, I. I. Mazin, L. Chioncel, and A. I. Lichtenstein, Physical Review B 67, 153106 (2003).

[58] V. I. Anisimov and F. Aryasetiawan, Journal of Physics: Condensed Matter 9, 767 (1997).

[59] V. I. Anisimov and O. Gunnarsson, Physical Review B 43, 7570 (1991).

[60] O. Bengone, M. Alouani, P. Blöchl, and J. Hugel, Physical Review B 62, 16392 (2000).

[61] A. Rohrbach, J. Hafner, and G. Kresse, Physical Review B 70, 125426 (2004).
[62] M. Cococcioni and S. de Gironcoli, Physical Review B 71, 035105 (2005).

[63] M. K. Drulis, H. Drulis, S. Gupta, M. W. Barsoum, and T. El-Raghy, Journal of Applied Physics 99, 093502 (2006).

[64] S. E. Lofland, J. D. Hettinger, K. Harrell, P. Finkel, S. Gupta, M. W. Barsoum, and G. Hug, Applied Physics Letters 84, 508 (2004).

[65] J. Wang and Y. Zhou, Physical Review B 69, 214111 (2004).

[66] J. D. Hettinger, S. E. Lofland, P. Finkel, T. Meehan, J. Palma, K. Harrell, S. Gupta, A. Ganguly, T. El-Raghy, and M. W. Barsoum, Physical Review B 72, 115120 (2005).

[67] B. Manoun, R. P. Gulve, S. K. Saxena, S. Gupta, M. W. Barsoum, and C. S. Zha, Physical Review B 73, 024110 (2006).

[68] J. C. Schuster, V. H. Nowotny, and C. Vaccaro, Journal of Solid State Chemistry 32, 213 (1980).

[69] A. Bouhemadou, Applied Physics A 96, 959 (2009).

[70] W. Zhou, L. Liu, and P. Wu, Journal of Applied Physics 106, 033501 (2009).

[71] W. Jeitschko, V. H. Nowotny, and F. Benesovsky, Monatshefte für Chemie und verwandte Teile anderer Wissenschaften 94, 672 (1963).

[72] C. S. Lue, J. Y. Lin, and B. X. Xie, Physical Review B 73, 035125 (2006).

[73] W. Jeitschko, V. H. Nowotny, and F. Benesovsky, Monatshefte für Chemie und verwandte Teile anderer Wissenschaften 94, 844 (1963).

[74] B. Manoun, S. Amini, S. Gupta, S. K. Saxena, and M. W. Barsoum, Journal of Physics: Condensed Matter 19, 456218 (2007).

[75] N. A. Phatak, S. R. Kulkarni, V. Drozd, S. K. Saxena, L. Deng, Y. Fei, J. Hu, W. Luo, and R. Ahuja, Journal of Alloys and Compounds 463, 220 (2008).

[76] S. Amini, A. Zhou, S. Gupta, A. DeVillier, P. Finkel, and M. W. Barsoum, Journal of Materials Research 23, 2157 (2011).

[77] M. K. Drulis, H. Drulis, A. E. Hackemer, O. Leaffer, J. Spanier, S. Amini, M. W. Barsoum, T. Guilbert, and T. El-Raghy, Journal of Applied Physics 104, 023526 (2008).

[78] M. W. Barsoum, T. H. Scabarozi, S. Amini, J. D. Hettinger, and S. E. Lofland, Journal of the American Ceramic Society 94, 4123 (2011).

[79] J. Etzkorn, M. Ade, D. Kotzott, M. Kleczek, and H. Hillebrecht, 182, 995 (2009).

[80] B. Manoun, S. Kulkarni, N. Pathak, S. K. Saxena, S. Amini, and M. W. Barsoum, Journal of Alloys and Compounds 505, 328 (2010).

[81] V. J. Keast, S. Harris, and D. K. Smith, Physical Review B 80, 214113 (2009).

[82] A. Thore, M. Dahlqvist, B. Alling, and J. Rosén, Computational Materials Science 91, 251 (2014).

[83] E. N. Caspi, P. Chartier, F. Porcher, F. Damay, and T. Cabioch, Materials Research Letters 3, 100 (2015).

[84] M. Dahlqvist, B. Alling, I. A. Abrikosov, and J. Rosén, Physical Review B 81, 024111 (2010).

[85] B. Anasori, M. Dahlqvist, J. Halim, E. J. Moon, J. Lu, B. C. Hosler, E. N. Caspi, S. J. May, L. Hultman, P. Eklund, J. Rosén, and M. W. Barsoum, Journal of Applied Physics 118, 094304 (2015).

[86] R. Meshkian, A. S. Ingason, M. Dahlqvist, A. Petruhins, 
U. B. Arnalds, F. Magnus, J. Lu, and J. Rosén, physica status solidi (RRL) - Rapid Research Letters 9, 197 (2015).

[87] C. C. Lai, R. Meshkian, M. Dahlqvist, J. Lu, L. A. Näslund, O. Rivin, E. N. Caspi, O. Ozeri, L. Hultman, P. Eklund, M. W. Barsoum, and J. Rosén, Acta Materialia 99, 157 (2015).

[88] O. Hellman, I. A. Abrikosov, and S. I. Simak, Physical Review B 84, 180301 (2011).

[89] P. Steneteg, B. Alling, and I. A. Abrikosov, Physical Review B 85, 144404 (2012).

[90] F. Rivadulla, M. Bañobre-López, C. X. Quintela, A. Piñeiro, V. Pardo, D. Baldomir, M. A. López-Quintela, J. Rivas, C. A. Ramos, H. Salva, J.-S. Zhou, and J. B. Goodenough, Nature Materials 8, 947 (2009).

[91] B. Alling, T. Marten, and I. A. Abrikosov, Nature Materials 9, 283 (2010).

[92] B. L. Gyorffy, A. J. Pindor, J. Staunton, G. M. Stocks, and H. Winter, Journal of Physics F: Metal Physics 15, 1337 (1985).

[93] A. Zunger, S.-H. Wei, L. G. Ferreira, and J. E. Bernard, Physical Review Letters 65, 353 (1990).

[94] A. Georges, G. Kotliar, W. Krauth, and M. J. Rozenberg, Reviews of Modern Physics 68, 13 (1996).

[95] F. Mercier, O. Chaix-Pluchery, T. Ouisse, and D. Chaussende, Applied Physics Letters 98, 081912 (2011).

[96] T. Goto and T. Hirai, Materials Research Bulletin 22, 1195 (1987).

[97] J. P. Palmquist, U. Jansson, T. Seppänen, P. O. A. Persson, J. Birch, L. Hultman, and P. Isberg, Applied Physics Letters 81, 835 (2002).

[98] P. Eklund, M. Beckers, U. Jansson, H. Högberg, and L. Hultman, Thin Solid Films 518, 1851 (2010).

[99] Q. M. Wang, A. F. Renteria, O. Schroeter, R. Mykhaylonka, C. Leyens, W. Garkas, and M. t. Baben, Surface and Coatings Technology 204, 2343 (2010).

[100] T. Gebhardt, D. E. Hajas, M. Scholz, B. Hallstedt, B. Cappi, J. Song, R. Telle, and J. M. Schneider, Materials Science and Engineering: A 525, 200 (2009).

[101] K. Buchholt, R. Ghandi, M. Domeij, C. M. Zetterling, J. Lu, P. Eklund, L. Hultman, and A. L. Spetz, Applied Physics Letters 98, 042108 (2011).

[102] J. Rosén, L. Ryves, P. O. Å. Persson, and M. M. M. Bilek, Journal of Applied Physics 101, 056101 (2007).

[103] H. Högberg, L. Hultman, J. Emmerlich, T. Joelsson,
P. Eklund, J. M. Molina-Aldareguia, J. P. Palmquist, O. Wilhelmsson, and U. Jansson, Surface and Coatings Technology 193, 6 (2005).

[104] J. P. Palmquist, S. Li, P. O. Å. Persson, J. Emmerlich, O. Wilhelmsson, H. Högberg, M. Katsnelson, B. Johansson, R. Ahuja, O. Eriksson, L. Hultman, and U. Jansson, Physical Review B 70, 165401 (2004).

[105] R. J. Weiss, Proceedings of the Physical Society 82, 281 (1963).

[106] M. van Schilfgaarde, I. A. Abrikosov, and B. Johansson, Nature 400, 46 (1999).

[107] A. Mockute, P. O. Å. Persson, J. Lu, A. S. Ingason, F. Magnus, S. Olafsson, L. Hultman, and J. Rosén, Journal of Materials Science 49, 7099 (2014).

[108] M. Ziese, I. Vrejoiu, and D. Hesse, Applied Physics Letters 97, 052504 (2010).

[109] L. Farber and M. W. Barsoum, Journal of Materials Research 14, 2560 (1999).

[110] M. Junaid, C. L. Hsiao, J. Palisaitis, J. Jensen, P. O. Å. Persson, L. Hultman, J. Birch, and J. Birch, Applied Physics Letters 98, 141915 (2011).

[111] L. M. Corliss, J. M. Hastings, and R. J. Weiss, Physical Review Letters 3, 211 (1959).

[112] R. Hafner, D. Spišák, R. Lorenz, and J. Hafner, Physical Review B 65, 184432 (2002).

[113] P. Rhodes and E. P. Wohlfarth, Proceedings of the Royal Society of London A: Mathematical, Physical and Engineering Sciences 273, 247 (1963).

[114] E. P. Wohlfarth, Journal of Magnetism and Magnetic Materials 7, 113 (1978).

[115] J. Kübler, Theory of Itinerant Electron Magnetism (OUP Oxford, 2009).

[116] T. Chatterji, Neutron Scattering from Magnetic Materials (Gulf Professional Publishing, 2005).

[117] P. Eklund, T. Cabioch, V. Mauchamp, M. Jaouen, and M. W. Barsoum, Journal of the European Ceramic Society 33, 897 (2013).

[118] R. Burriel, L. Tocado, E. Palacios, T. Tohei, and H. Wada, Journal of Magnetism and Magnetic Materials 290-291, 715 (2005).

[119] C. Hu, C. C. Lai, Q. Tao, J. Lu, J. Halim, L. Sun, J. Zhang, J. Yang, B. Anasori, J. Wang, Y. Sakka, L. Hultman, P. Eklund, J. Rosén, and M. W. Barsoum, Chemical Communications 51, 6560 (2015).

[120] M. Naguib, V. N. Mochalin, M. W. Barsoum, and Y. Gogotsi, Advanced Materials 26, 992 (2013).

[121] M. Naguib, M. Kurtoglu, V. Presser, J. Lu, J. Niu, M. Heon, L. Hultman, Y. Gogotsi, and M. W. Barsoum, Advanced Materials 23, 4248 (2011). 